\title{
Solubility of spruce galactoglucomannans determines interfacial morphology and emulsion stability
}

\section{Mamata Bhattarai ( $\nabla$ mamata.bhattarai@helsinki.fi )}

University of Helsinki: Helsingin Yliopisto https://orcid.org/0000-0001-7121-4602

Inkeri Kontro

University of Helsinki: Helsingin Yliopisto

Irina Sulaeva

BOKU: Universitat fur Bodenkultur Wien

Fabio Valoppi

University of Helsinki: Helsingin Yliopisto

Antje Potthast

BOKU: Universitat fur Bodenkultur Wien

Kirsi S. Mikkonen

University of Helsinki: Helsingin Yliopisto

\section{Research Article}

Keywords: polysaccharide solubility, wood hemicelluloses, colloidal particles, emulsions, emulsion interface

Posted Date: February 16th, 2022

DOI: https://doi.org/10.21203/rs.3.rs-1336559/v1

License: (9) This work is licensed under a Creative Commons Attribution 4.0 International License.

Read Full License 
1 Solubility of spruce galactoglucomannans determines interfacial morphology and 2 emulsion stability

3 Mamata Bhattarai $^{1^{*}}$, Inkeri Kontro ${ }^{2}$, Irina Sulaeva ${ }^{3}$, Fabio Valoppi $^{1,4}$, Antje Potthast ${ }^{3}$, Kirsi S. Mikkonen ${ }^{1,4}$

4

Dr. Mamata Bhattarai

Department of Food and Nutrition, P.O. Box 66, 00014 University of Helsinki, Finland mamata.bhattarai@helsinki.fi

Dr. Inkeri Kontro

Department of Physics, P.O. Box 64, 00014 University of Helsinki, Finland inkeri.kontri@helsinki.fi

Dr. Irina Sulaeva

Department of Chemistry, University of Natural Resources and Life Sciences, Konrad-Lorenz-Straße. 24, 3430 Tulln, Austria irina.sulaeva@boku.ac.at

Dr. Fabio Valoppi

Department of Food and Nutrition, P.O. Box 66, 00014 University of Helsinki, Finland

Helsinki Institute of Sustainability Science, University of Helsinki, P.O. Box 65, 00014 Finland fabio.valoppi@helsinki.fi

Prof. Antje Potthast

Department of Chemistry, University of Natural Resources and Life Sciences, Konrad-Lorenz-Straße. 24, 3430

Tulln, Austria

antje.potthast@boku.ac.at

Prof. Kirsi S. Mikkonen

Department of Food and Nutrition, P.O. Box 66, 00014 University of Helsinki, Finland Helsinki Institute of Sustainability Science, University of Helsinki, P.O. Box 65, 00014 Finland kirsi.s.mikkonen@helsinki.fi

\section{Abstract}

Specific supramolecular interactions in polysaccharides change their solubility rendering diverse interfacial properties in oil-in-water $(\mathrm{O} / \mathrm{W})$ emulsions. We studied the effect of solubility of softwood hemicelluloses, spruce galactoglucomannans (GGMs) isolated by pressurized hot-water extraction methods or recovered as side streams in a thermomechanical pulp (TMP) mill on interfacial structures and stability of $\mathrm{O} / \mathrm{W}$ emulsions. Detailed characterization of GGMs revealed a soluble (molecularly dispersed) fraction of molar mass $8.9 \times 10^{3}-2.1 \times 10^{4} \mathrm{~g}$ $\mathrm{mol}^{-1}$ and sub-micron-sized insoluble fractions that were either loose assemblies or fractal type supramolecular aggregates and agglomerates. Based on the relative share of these fractions and nanostructural complexity, GGMs were classified into soluble, semi-soluble, and insoluble. GGMs extracted via pressurized hot-water extraction methods were soluble to semi-soluble compared to GGMs recovered from the TMP process. Semi-soluble GGMs exhibited efficient stability of dispersed oil droplets in emulsions followed by insoluble and soluble GGMs. With an increasing share of insoluble fractions and their structural complexity, emulsion's interfacial morphology changed from smooth to diffused type. Comparing GGMs with soluble small-molecule surfactant Tween 20 and insoluble alkali extracted beechwood glucuronoxylans, our findings suggest that surface activity of adsorbing 
soluble or insoluble fraction dominates the interfacial morphology. Under the condition of sufficient interfacial coverage, insoluble fractions complement the emulsion stability with a filling effect in the continuous phase of emulsions. The findings improve our understanding of bio-based polysaccharides' solubility, their emulsion stability mechanisms, and strategies to tailor via biorefining approaches.

Keywords: polysaccharide solubility; wood hemicelluloses; colloidal particles; emulsions; emulsion interface

\section{Introduction}

Stabilization of dispersed systems such as emulsions by biopolymers and bio-based colloidal particles is an emerging area of research in life science applications, including food and pharmaceuticals (Dickinson 2003). Polysaccharides are an important class of biopolymers obtained from plants, microbes, seaweed, and algae, and utilized with or without physical/chemical modification (Dickinson 2003). Their key functions as emulsifiers, stabilizers, or gelators are governed by solubility, which is a result of complex inter-/intramolecular interactions between inherent characteristics of polysaccharides (molar mass, degree of branching/substitution, functional groups, monosaccharide composition), solvent and solvent environment ( $\mathrm{pH}$, ionic strength, temperature) (Whistler 1973). When soluble, polysaccharides attain a molecular dispersed state with individual molecules commonly in a random coil conformation, while insoluble polysaccharides display colloidal particle behavior. Cellulose and chitin are examples of the latter. Molecular associations result from H-bonding, van der Waals and/or hydrophobic interactions between functional groups in polysaccharides, e.g., hydroxyl, acetyl, carbonyl groups (Dumitriu 2004) or covalently bound non-polysaccharide components such as bound proteins or phenolic groups, e.g., proteins in gum Arabic (Sanchez et al. 2018), ferulic acid in pectins (Zhang et al. 2015), and arabinoxylans (Ebringerova et al. 1994).

Solubility affects the interfacial organization of polysaccharides in oil-in-water $(\mathrm{O} / \mathrm{W})$ emulsions. Soluble polysaccharides such as gum Arabic and pectins stabilize the oil-water interface via steric stabilization mechanism from the adsorption of surface-active polysaccharide molecules and formation of a viscoelastic layer at the oilwater interface (Nakauma et al. 2008). Insoluble polysaccharides such as cellulose and chitins with particle-like characteristics have reported Pickering-type stabilization (Lam et al. 2014). In addition, both soluble and insoluble polysaccharides stabilize the dispersed state via viscosity-enhancing effect and/or form a three-dimensional colloidal network in the continuous phase (Dickinson 2017).

In the present work, we demonstrate the effect of polysaccharide solubility on emulsion stability in the context of wood hemicelluloses. Wood hemicelluloses constitute about $15-25 \mathrm{wt} \%$ of dry wood cells however, they are 
underutilized and understudied than other wood biopolymers. They have gained popularity in recent years because of their abundance and potential to replace fossil-derived materials. Galactoglucomannans (GGMs) are predominant hemicelluloses in softwood and are composed of partially acetylated $\beta$-( $1 \rightarrow 4)$-linked mannopyranosyl and $\beta$-( $1 \rightarrow 4)$-linked glucopyranosyl chains substituted by $\alpha-(1 \rightarrow 6)$-linked galactopyranosyl units with an average degree of acetylation is 0.3(Sjöström 1993). Spruce GGMs have demonstrated promising emulsifying and stabilizing properties in $\mathrm{O} / \mathrm{W}$ emulsions, more efficiently than commonly used hydrocolloids, gum Arabic, and corn fiber gum (Bhattarai et al. 2019; Lehtonen et al. 2016; Mikkonen et al. 2016b). Lignins and lignin-derived phenolic compounds, often considered impurities, are ascribed to the stabilizing performance of GGMs. However, the macromolecular state of GGMs in an aqueous solution and eventually at the oil-water interface is largely unknown. We have previously noted that GGMs isolated from pressurized hot water extraction (PHWE) were semi-soluble and solubility depended on the purity of the extracts. There were evidences of supramolecular aggregates, agglomerates, and colloidal particles(Bhattarai et al. 2020) suggesting the need to investigate the multiphase emulsion system stabilized by GGMs by comprehensively studying their solubility.

In this study, we evaluate the solubility of GGMs recovered as industrial side-streams from the thermomechanical pulping process (TMP) and hot water extraction of wood chips using the BLN process (named after inventors) (Schoultz 2015). Recovered GGMs from these industrially relevant extraction approaches have previously exhibited well to satisfactory emulsion stabilization abilities. These GGMs differ in molar mass and total content of phenolic compounds; therefore, we hypothesize these affect their solubility. To understand the effect of recovery processes on GGM solubility, we compare these results with our recent findings on GGMs obtained from the PHWE process (Fig. 1). In the next step, we characterize the oil-water interface stabilized by GGMs and evaluate emulsion stability during 2-week storage. Solubility and emulsion stabilization mechanism of GGMs are compared to glucuronoxylans from beechwood (BGX), a hardwood species, recovered via alkali-extraction known to form insoluble particles (Bosmans et al. 2014; Linder et al. 2003) and soluble nonionic surfactant Tween 20 (T20) currently used as emulsifiers in food and pharmaceutical industries.

This study contributes to our understanding of the colloidal properties of polysaccharides, especially those from plant extracts with varying purity, and the possibility to tailor an application-based recovery approach to isolate them. As the demand for bio-based hydrocolloids is rising with an emphasis given to those obtained from unused/waste resources, knowledge on potentially valuable wood hemicelluloses will facilitate their future use in pharmaceuticals, food, and cosmetics sectors, for example. 

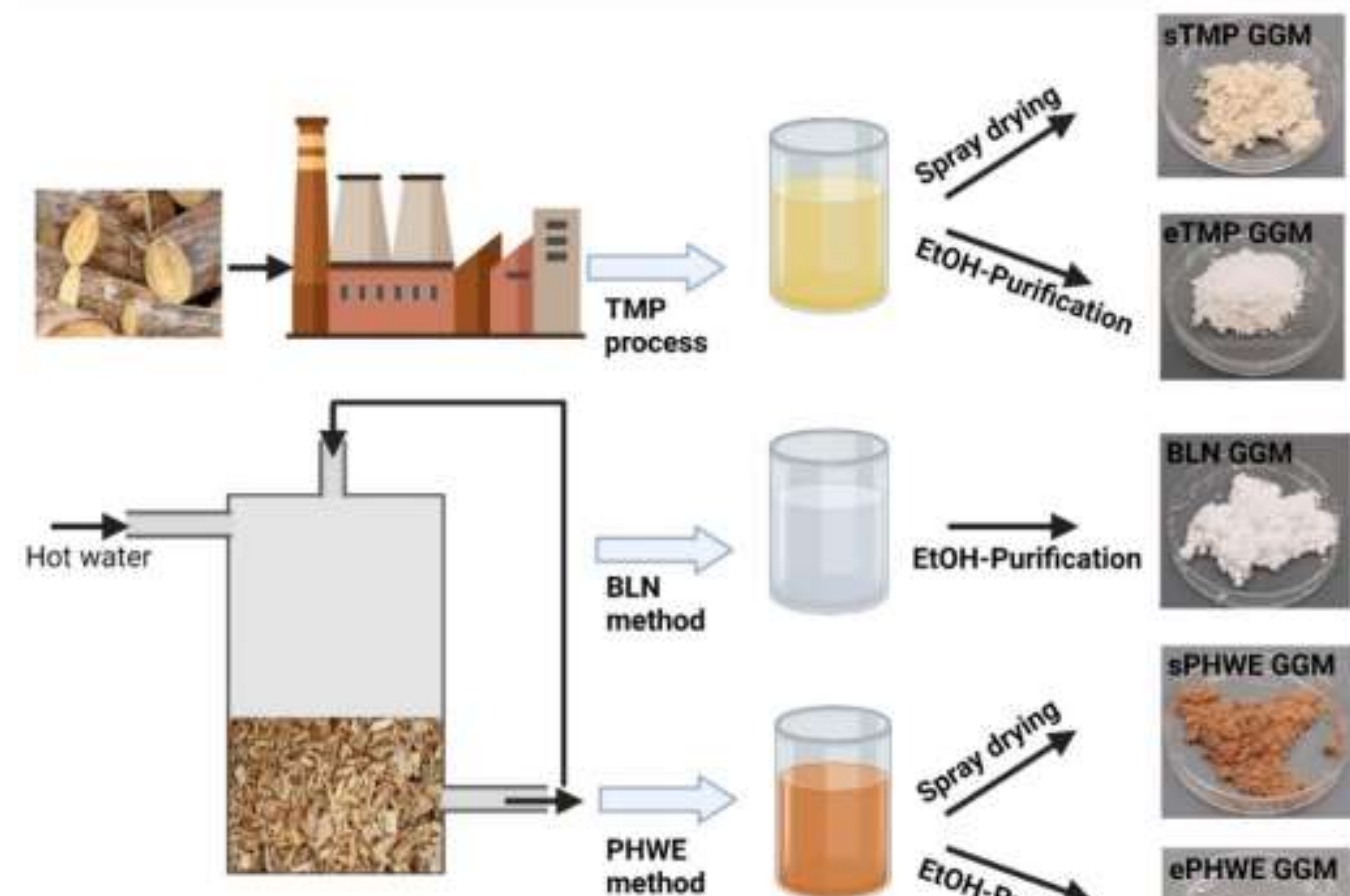

method
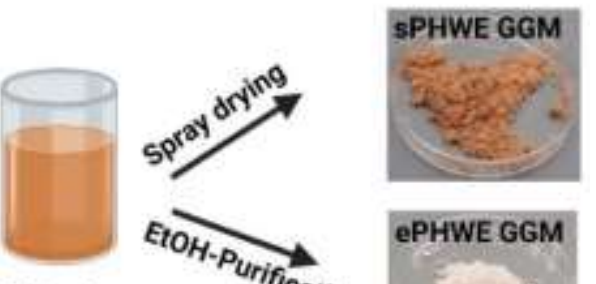

\section{PHWE}

Extracts

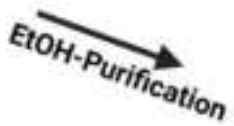

ePHWE GGM

Fig.1 Schematic illustration of GGMs recovery processes

\section{Experimental Section}

\subsection{Chemicals}

108 BGX was supplied in a powdered form from Sigma-Aldrich (St. Louis, MO, USA), now Merck. T20 was from

109 VWR Chemicals (Leuven, Belgium). Rapeseed oil purchased from a local supermarket was from Bunge Finland

110 Oy (Raisio, Finland). Citric acid monohydrate, sodium azide, and sodium hydroxide used for asymmetric flow

111 field-flow fractionation (AF4) experiments were from Merck (Darmstadt, Germany). For other experiments,

112 sodium hydroxide was from VWR Chemicals.

\section{$113 \quad 2.2$ Hemicelluloses isolation}

114 GGMs were recovered from Norway spruce (Picea abies) using three different isolation methods: as an industrial

115 side stream and from two different types of hot water extractions of wood chips/sawdust.

116 GGMs were recovered from the TMP process in a Finnish TMP mill on an industrial-scale following the method

117 of Willför et al. (2003) Mechanical shearing during the TMP process releases water-soluble hemicelluloses from

118 wood into the process water,(Thornton 1994; Willför et al. 2003) which was collected after a series of filtration

119 and ultra-filtration. The resulting isolate was used after spray drying and after its purification using ethanol added 
122 Another GGMs was obtained by hot water extraction namely, BLN method patented by Schoultz (2015) and

123 PHWE method based on Kilpeläinen et al. (2014) Water at $150-170{ }^{\circ} \mathrm{C}$ was used to recover GGMs from wood

124 chips (BLN) or sawdust (PHWE) in a flow-through extractor. Using the PHWE method, the extract was collected

125 for 70 min whereas using the BLN method, the extract was recirculated multiple times through the biomass before

126 collection. By doing so, aromatic residues in the extract such as lignins, lignin-derived compounds, and extractives

127 were readsorbed back to the biomass to obtain high purity. The extract was further purified by adding ethanol at

$1283: 1 \mathrm{v} / \mathrm{v}$ (ethanol: extract) and allowed to precipitate. The pellet was collected after centrifugation, washed further

129 with ethanol, and dried in a vacuum oven at $40{ }^{\circ} \mathrm{C}$. This GGM sample is referred to as BLN GGM. The extract

130 from the PHWE process was used after spray-drying(Valoppi et al. 2019) referred to as sPHWE GGM and after

131 purification using ethanol was added to the extract at $8: 1$ followed by drying in a vacuum oven at $40{ }^{\circ} \mathrm{C}$, referred

132 to as ePHWE GGM.

133 GGMs recovered using different biorefining approaches varied in the content of polysaccharides, phenolic

134 compounds, and extractives (Table 1) (Mikkonen et al. 2019). BGX was primarily $\beta$-(1 $\rightarrow 4$ )-linked xylopyranosyl

135 units with $\geq 90 \%$ purity, according to the manufacturer.

136 Table 1. Types of GGM samples included in the study. Total polysaccharide content wt $\%$, phenolic, and extractive 137 content of samples according to Mikkonen et al. (2019).

\begin{tabular}{lllll}
\hline Samples & Recovery approach & $\begin{array}{l}\text { Polysaccharide } \\
\text { content wt\% }\end{array}$ & $\begin{array}{l}\text { Phenolic content } \\
{[\mathbf{m g} / \mathbf{g}]^{\mathbf{b})}}\end{array}$ & $\begin{array}{l}\text { Extractives } \\
{[\mathbf{m g} / \mathbf{g}]^{\mathbf{c}}}\end{array}$ \\
\hline sTMP GGM & $\begin{array}{l}\text { Spray-dried recovered from TMP } \\
\text { process (Unpurified) }\end{array}$ & 66 & 19.4 & 9.1 \\
eTMP GGM & $\begin{array}{l}\text { Ethanol-precipitated recovered } \\
\text { from TMP process (Purified) }\end{array}$ & 73 & 5.1 & 0.89 \\
BLN GGM & $\begin{array}{l}\text { Ethanol-precipitated extracted } \\
\text { using BLN method (Purified) }\end{array}$ & 79.5 & 2.3 & 0.14 \\
sPHWE GGM & $\begin{array}{l}\text { Spray-dried extracted using } \\
\text { PHWE method (Unpurified) }\end{array}$ & 71 & 48.7 & 5.1 \\
ePHWE GGM & $\begin{array}{l}\text { Ethanol-precipitated extracted } \\
\text { using PHWE process (Purified) }\end{array}$ & 78.4 & 15.8 & 0.14 \\
\hline
\end{tabular}

a) Total polysaccharide content determined by summing up monosaccharides (mg/g) of dry GGM samples (excluding free monosaccharides) after applying correction factors 


\subsection{Aqueous phases preparation and characterization}

144 Aqueous phases of wood hemicelluloses were prepared at $1 \%(\mathrm{w} / \mathrm{v})$ in $25 \mathrm{mM}$ sodium citrate buffer at $\mathrm{pH} 4.5 \mathrm{by}$ 145 mixing overnight with a magnetic stirrer at room temperature $(\mathrm{RT})\left(22-23{ }^{\circ} \mathrm{C}\right)$. The aqueous phases were also 146 studied after high-intensity mechanical shearing treatment, where they were mechanically mixed at $11,000 \mathrm{rpm}$ 147 for 5 min using Ultra-Turrax (T-18 basic, IKA, Staufen, Germany) followed by three passes in a microfluidizer 148 (Microfluidizer 110Y, Microfluidics, Westwood, MA, USA) at 800-850 bar. The aqueous phases were 149 characterized using the following techniques.

\section{$150 \quad$ 2.3.1 AF4-MALS experiments}

151 To evaluate solubility, soluble and insoluble fractions were separated using AF4. In this flow-based separation 152 technique, differently sized fractions are first segregated (also known as focusing) based on their hydrodynamic 153 size on a membrane. Next, during the elution, the fractions are eluted based on descending size order. For a high 154 separation resolution, cross-flow perpendicular to the main flow is applied (Podzimek 2011). AF4 coupled with 155 MALS and dRI detectors provides comprehensive information on the size and shape characteristics of separated 156 fractions in a non-invasive way and without the compulsion of pre-filtration of samples. In our latest study, we 157 employed this method to evaluate the solubility of sPHWE GGM and ePHWE GGM (Bhattarai et al. 2020). 158 Detailed information about the AF4 setup, method optimization, refractive index increment $(d n / d c)$ measurement, 159 MALS data collection, and evaluation have been described in the study. In the present study, using the same 160 method, we characterized the aqueous phases of other samples: sTMP GGM, BLN GGM, and BGX. The eTMP 161 GGM had some insoluble particles; therefore, it was not studied to avoid the risk of particle sedimentation in the 162 detector cells and blockage in connecting capillary tubes.

163 Aqueous phases were injected as such and after filtration through a $0.2 \mu \mathrm{m}$ syringe filter. The injection volume 164 was $100 \mu \mathrm{L}$ and $200 \mu \mathrm{L}$ for unfiltered and filtered samples, respectively. Autosampler Agilent G1313A and Agilent G1213A were used for the $100 \mu \mathrm{L}$ and $200 \mu \mathrm{L}$ injection, respectively. Samples were focused for $10 \mathrm{~min}$

166 on a regenerated cellulose Ultracel membrane from Merck KGaA (Darmstadt, Germany) with a molecular weight cutoff of $3 \mathrm{kDa}$. For a better size separation during the elution, a declining crossflow rate $\left(\mathrm{V}_{\mathrm{x}}\right)$ starting from 3

$168 \mathrm{mLmin}^{-1}$ was applied for each sample, which is presented with the AF4 eluograms in Fig. 2.

169 An online combination of Wyatt DAWN HELEOS II MALS detector and a Wyatt TRex dRI detector, both from 170 Wyatt Technologies, Santa Barbara, USA was used in series for the macromolecular analysis of separated fractions.

171 All detectors were set at $25^{\circ} \mathrm{C}$, whereas the separation channel was at RT $\left(22-23^{\circ} \mathrm{C}\right)$. 
173 data evaluation was performed by software Astra 6.1 (Wyatt Technologies, Santa Barbara, USA). Exponential fit

174 with fit order 1-4 was used to obtain molar mass values of the separated fraction. The total mass recovery was

175 calculated by the software, which considers the dRI calibration constant, injected mass, $d n / d c$ value, elution time,

176 and flow rate. The sample recovery was obtained from the unfiltered samples. The mass recovery percentage of each fraction presented in Fig. 2 is normalized by the total sample recovery (Table S1, supplementary data).

\section{$178 \quad$ 2.3.2 Small-angle X-ray scattering (SAXS) on aqueous phases}

179 SAXS experiments were performed on aqueous phases of hemicelluloses with and without shear treatment at

180 Diamond Light Source Synchrotron (Didcot, Oxfordshire, UK) with the standard solution SAXS setup of beamline

181 B21 (bioSAXS robot) at $20^{\circ} \mathrm{C}$. The experimental details have been reported in our previous study(Bhattarai et al.

182 2020). Briefly, the photon wavelength $(\lambda)$ was $0.1 \mathrm{~nm}$. The scattering vector $q$ defined as $q=\frac{4 \pi \sin \theta}{\lambda}$, where $\theta$ is

183 half of the scattering angle related to distances in real space by $d=\frac{2 \pi}{q}$. The obtained $q$-range was 0.032 to $3.8 \mathrm{~nm}^{-}$

$184{ }^{1}$ and the measured sample volume was $35 \mu \mathrm{L}$. The measurement of shear-treated samples was performed after $4-$

1855 days of treatment, as they had to be shipped to the synchrotron facility. During this time, samples were stored at

186 RT. To understand the effect of heat and freezing at the nanostructure, selected GGM samples were heated to

$18770{ }^{\circ} \mathrm{C}$ for $3 \mathrm{~min}$, frozen at $-80^{\circ} \mathrm{C}$ for $30 \mathrm{~min}$. Samples were kept at RT before the SAXS measurement.

188 Scattering features in the aqueous phases were examined and power-law was fitted when scattering intensity $(I)$

189 would exhibit such behavior at sufficiently long $q$-range. The power law is given by:

$$
I(q) \propto q^{-p}
$$

Equation 1

191 where $p$ is the power-law coefficient. A $p<3$ indicates structures described as mass fractals (e.g., wrinkled paper).

192 A $p$-value between 3 and 4 arises from surface fractals with a dense and homogenous inner structure, whereas $p=$

1934 indicates smooth and well-defined interfaces. A special case of $p=1$ and $p=2$ correspond to thin cylinders or

194 strings and thin planes or disks, respectively (Schmidt 1991).

\section{$195 \quad$ 2.3.3 Zeta potential analysis}

196 The zeta potential was measured at $25^{\circ} \mathrm{C}$ in a zeta sizer (Zetasizer Nano ZS, Malvern Instruments) with a $633 \mathrm{~nm}$

197 standard laser and $173^{\circ}$ backscattering angle (supplementary data, Table S4) according to Bhattarai et al. (2019)

198 The measurement was performed on shear-treated samples within 1-2 days of the treatment. 


\subsection{Emulsion preparation and characterization}

200 In the second part of the study, emulsions were prepared using the GGMs. All emulsions were prepared using 1 $201 \mathrm{wt} \%$ hemicellulose and $2.5 \mathrm{wt} \%$ rapeseed oil to ensure an emulsifier-rich regime (Bhattarai et al. 2019; Mikkonen 202 et al. 2016b). Emulsions from sPHWE GGM and ePHWE GGM were also prepared with 0.5 wt $\%$ and 5 wt $\%$ oil 203 for their interfacial features characterization using SAXS (reported further). Emulsions were also prepared using $1 \mathrm{wt} \% \mathrm{BGX} / \mathrm{T} 20$ and $2.5 \mathrm{wt} \%$ oil to be included for comparison.

Emulsions were prepared in two steps following our previous protocol (Bhattarai et al. 2019). The aqueous phases with stabilizers and oil were first mechanically mixed using Ultra-Turrax followed by high-pressure homogenization using a microfluidizer under the same conditions mentioned in section 2.3. Emulsions were stored for 2 weeks at RT with $0.02 \mathrm{wt} \%$ sodium azide as a preservative against microbial spoilage.

\subsubsection{SAXS of emulsions}

210 The interfacial features in emulsions were characterized using SAXS. Measurements were performed after 4-5 211 days of preparation. Samples were stored at RT during this time. Selected emulsions were also studied after 212 thawing at RT after freezing to $-80^{\circ} \mathrm{C}$ and after 8 weeks of storage at RT.

213 The background-corrected scattering data were fitted using MATLAB (Math Works Inc., Massachusetts, USA) 214 with a model consisting of a linear combination of the one-dimensional scattering patterns of the backgroundcorrected aqueous phase, oil, and a power-law behavior given by:

$$
I_{f i t}(q)=A q^{-p}+B I_{o i l}+C I_{a q}
$$

Equation 2

217 where, $A, B, C$, and $p$ are constants, $q$ is the magnitude of the scattering vector, and $I_{o i l}$ and $I_{a q}$ are the measured 218 scattering intensities of oil and the aqueous phase, respectively. The error analysis was performed for the emulsions, 219 aqueous phase, and oil by Monte Carlo (MC) procedure, where 1000 data sets were generated based on SAXS 220 intensities. An example of data generated in the MC procedure is shown in Fig. S2B, supplementary data. The fits 221 were averaged and examined for outliers. Standard deviations of fitting parameters are reported as a fitting error 222 (Table S3, supplementary data).

\subsubsection{Cryo-scanning electron microscopy (Cryo-SEM)}

224 Based on SAXS experimental results, selected emulsions were visualized under cryo-SEM. A 2-3 $\mu$ L of emulsions 225 were sandwiched between two planchettes $(2 \times 100 \mu \mathrm{m})$ and cryopreserved by a Leica EM HPM100 high-pressure 226 freezer (Leica Microsystems GmbH, Wetzlar, Germany). The sandwiched planchettes were mounted in a 227 planchette holder under liquid nitrogen and transferred to Leica MED020 high-vacuum coating system (Leica 

samples were freeze-fractured, sublimated at $-90^{\circ} \mathrm{C}$ for $1 \mathrm{~min}$, and sputter-coated with Carbon/Platinum with a

230 thickness of $6 \mathrm{~nm}$. Specimens were examined with an FEI Quanta 3D SEM (ThermoFisher Scientific,

231 Massachusetts, USA) operated at an accelerating voltage of $2 \mathrm{kV}$ at approximately $-140{ }^{\circ} \mathrm{C}$.

\subsubsection{Droplet size distribution}

233 The droplet size distribution of emulsions was determined using a Mastersizer 3000 Hydro EV (Malvern

234 Instruments Ltd, Worcestershire, UK) according to our previous study (Bhattarai et al. 2019). Measurements were 235 performed on the emulsion preparation day, after 1, and 2 weeks of storage at RT. Droplet size is reported as 236 surface-average $\mathrm{D}(3,2)$ and volume-average $\mathrm{D}(4,3)$ (supplementary data, Fig. S3). The results are the mean and 237 standard error of the mean of at least three runs from each measurement $(\mathrm{n}=3)$.

\section{$238 \quad$ 3.1.4 Destabilization kinetics}

239 Creaming or sedimentation in emulsion mainly results from coalescence and/or flocculation of oil droplets. The 240 kinetics of these dynamic changes during emulsion storage was evaluated using a Turbiscan Lab expert 241 (Formulaction, Toulouse, France). A $20 \mathrm{~mL}$ emulsion was poured into a semi-flat bottom glass vial. Transmission 242 and backscattering (BS) signals from emulsions were obtained every day during 4-week storage at RT by scanning 243 the entire emulsion height rather than a specific height point. During this time, emulsions were stored unshaken. 244 After each scan, changes in transmission and BS signals from the previous scan were compared and analyzed by 245 the software Turbisoft version 1.2 (Formulaction, Toulouse, France) to obtain the Turbiscan stability index (TSI) 246 for each emulsion with higher TSI meaning greater changes in the emulsion.

247 To predict emulsion stability and kinetics of changes during prolonged storage, a non-linear regression was 248 performed on the TSI using the following equation:

$$
T S I=T S I_{\max } \cdot\left(1-e^{-k \cdot t}\right)
$$

Equation 3

250 where $T S I_{\max }$ is the maximum TSI reachable at infinite time, $k$ is the rate constant, and $t$ is time. The Levenberg251 Marquardt algorithm was used to perform a least-squares function minimization using GraphPad Prism v. 5.03 252 (GraphPad Software, San Diego, CA, USA). The goodness of fit was evaluated based on statistical parameters of 253 fitting $\left(\mathrm{R}^{2}, P\right.$-value, and standard error) and the residual analysis. To evaluate statistical differences among the 254 estimated regression parameters $\left(T S I_{\max }\right.$ and $k$ ) Bonferroni's Multiple Comparison test was performed. All 255 statistical analyses were performed at $P<0.05$. 


\section{Results and discussion}

\section{$257 \quad 3.1$ Solubility of aqueous hemicelluloses}

258 Separation of soluble and insoluble supramolecular fractions in aqueous hemicelluloses was performed by AF4

259 (Fig. 2), an upgraded technique to size-exclusion chromatography (SEC) traditionally used in polysaccharide 260 characterization. In AF4, separation occurs based on ascending order of hydrodynamic sizes during elution, 261 meaning, small-sized analytes elute ahead of larger ones. Quantitative estimation of separated analytes and their 262 absolute molar mass values are obtained from the differential refractive index (dRI) and multi-angle light scattering 263 (MALS) detectors' signals, respectively. Unlike SEC, sample filtration in AF4 is not mandatory and shear 264 degradation is minimal allowing the characterization of supramolecular fractions such as polysaccharide 265 aggregates and particles (Podzimek 2011). In our recent study, combining AF4 with MALS, complemented by 266 SAXS and transmission electron microscopy techniques, we were able to differentiate the molecularly dispersed 267 fraction, i.e., the soluble ones from the supramolecular i.e., insoluble fractions in hot-water extracted GGMs 268 (Bhattarai et al. 2020). Using a similar approach, we characterized GGMs recovered from TMP and BLN process 269 in this study.

270 The crude GGM extract from the TMP process (sTMP GGM) was highly polydisperse compared to GGM from 271 the BLN process (BLN GGM). The three dRI peaks and corresponding light scattering peaks represented three 272 major size fractions in sTMP GGM (Fig. 2B upper panel, smooth line) compared to only dRI peak in BLN GGM 273 (Fig. 2C upper panel). Upon filtration with $0.45 \mu \mathrm{m}$ pore size, the third fraction at higher retention times was 274 partially removed (Fig. 2B upper panel, dotted line). The BGX exhibited similar polydispersity, as sTMP GGM 275 except sample filtration did not affect the polydispersity (Fig. 2D upper panel). Using MALS data, the molar mass of each size fraction was obtained (Table S1). The molar mass of the first fraction in STMP GGM and BLN GGM 277 was $2.1 \times 10^{4} \mathrm{~g} \mathrm{~mol}^{-1}$ and $1.0 \times 10^{4} \mathrm{~g} \mathrm{~mol}^{-1}$, respectively, which was in agreement with previously reported for 278 GGMs (Mikkonen et al. 2016a). The molar mass of the first fraction of BGX was $1.5 \times 10^{4} \mathrm{~g} \mathrm{~mol}^{-1}$, also in 279 agreement with a previous study (Teleman et al. 2002). The values were also supported by the reported degree of 280 polymerization 100-200 of GGMs and glucuronoxylans in softwood and hardwood, respectively(Sjöström 1993) 281 all indicating that the first fraction constituted of soluble molecularly dispersed hemicelluloses. 

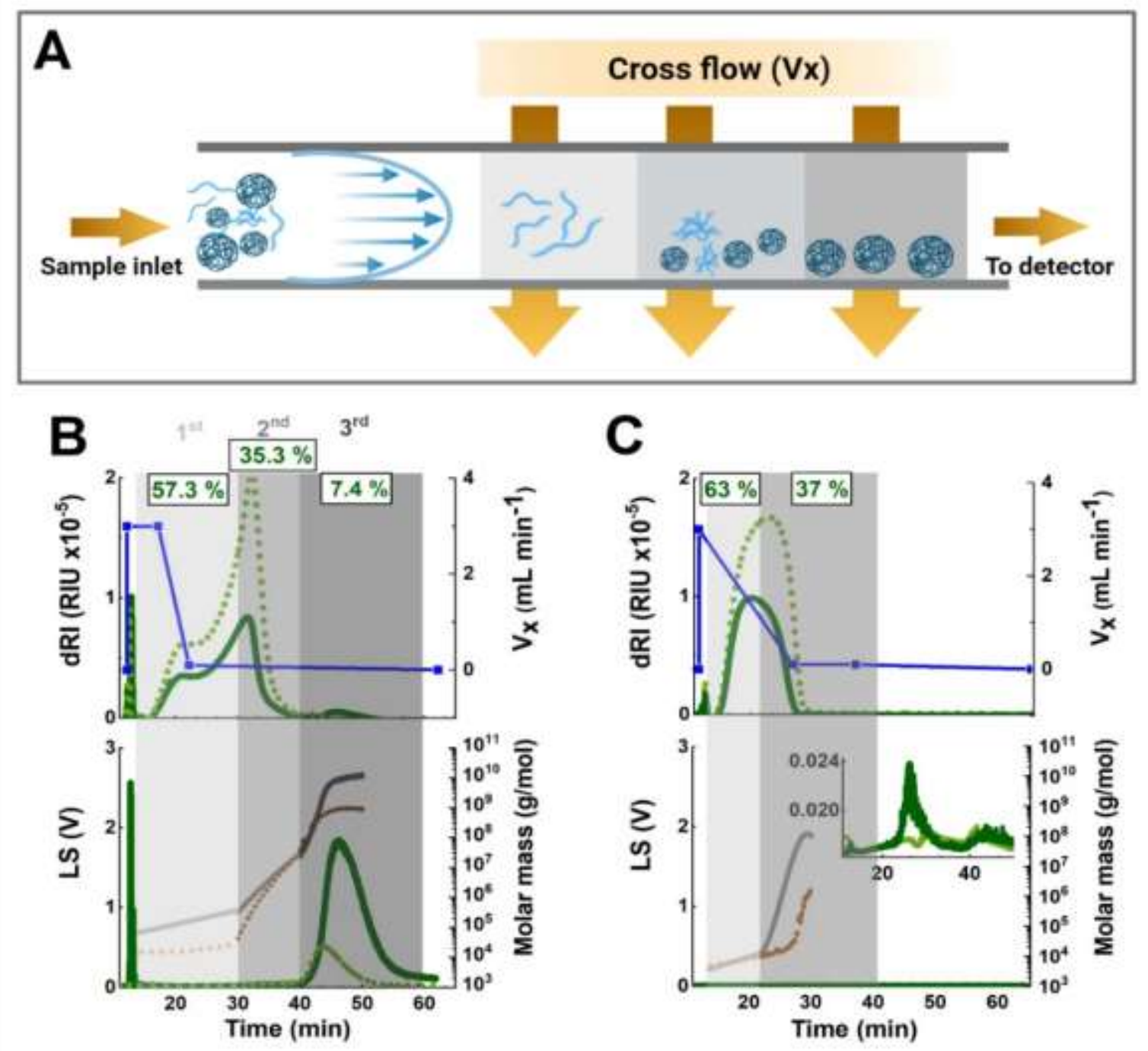

C

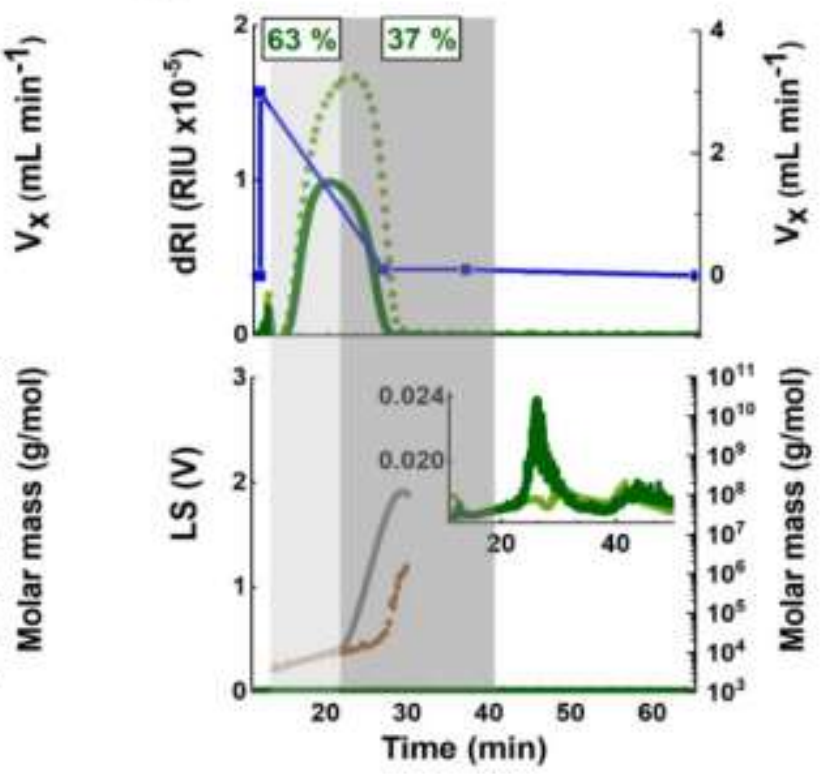

D

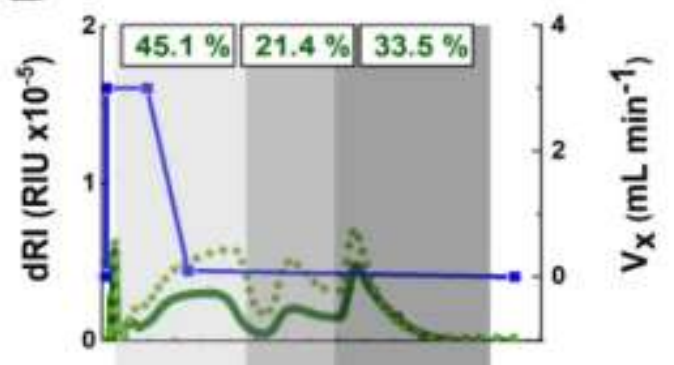

E
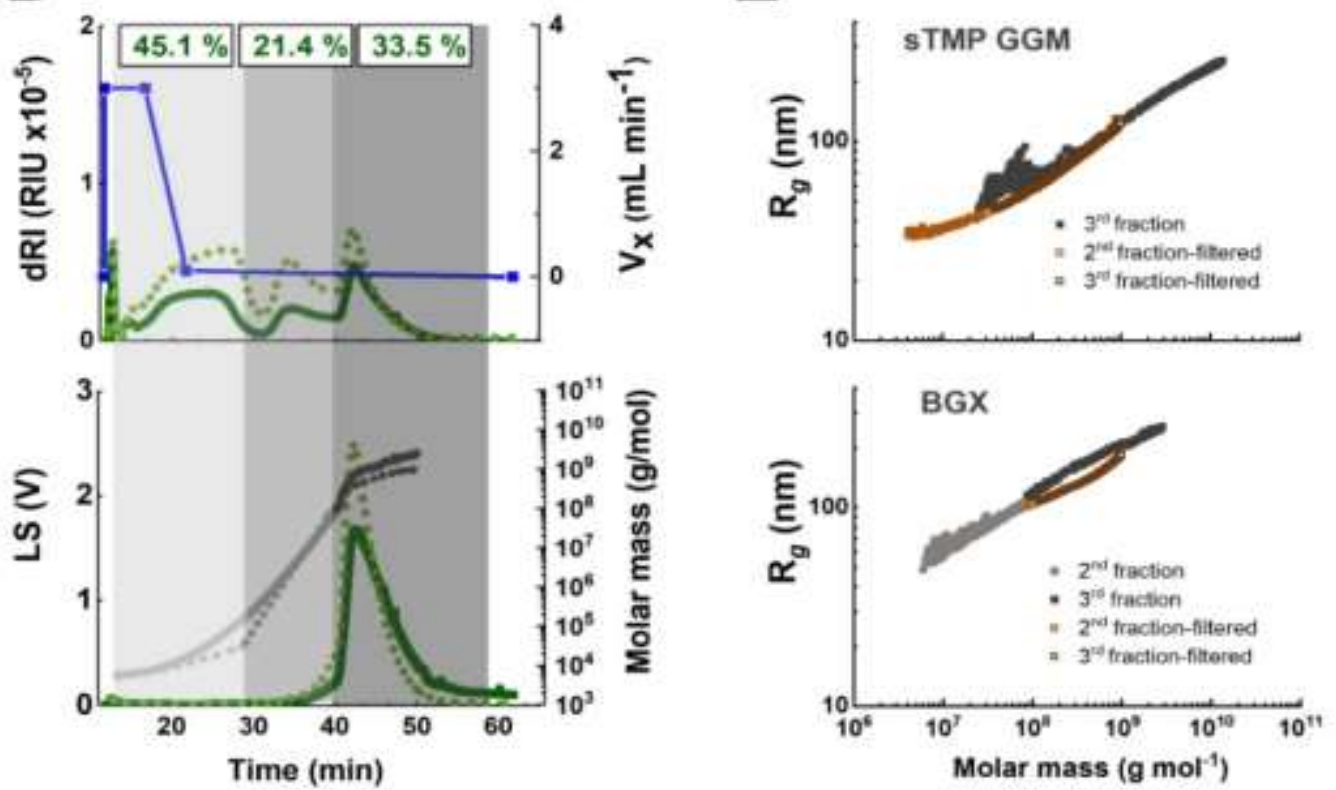

283 Fig. 2 (A) Schematic illustration of size-based fractionation of polydisperse samples in AF4. Differential refractive 
index (dRI) signal in refractive index units (RIU) and light scattering (LS) signal in volts during the AF4 elution of $1 \%$ (B) sTMP GGM, (C) BLN GGM, and (D) BGX in $25 \mathrm{mM}$ sodium citrate buffer at $\mathrm{pH} 4.5$ analyzed unfiltered (solid line) and filtered through a $0.2 \mu \mathrm{m}$ filter pore (dotted line). Applied crossflow rate $\left(\mathrm{V}_{\mathrm{x}}\right)$ during the elution presented with dRI signal (blue line with squares). Molar mass from unfiltered (solid line) and filtered (dotted line) of separated fractions, differentiated by color gradient and presented with LS signals. The recovery $\%$ of each fraction was calculated only for the unfiltered sample-based the dRI signal. (E) Log-log plot of the radius of gyration $\left(\mathrm{R}_{g}\right)$ versus the molar mass of second $\left(2^{\text {nd }}\right)$ and third $\left(3^{\text {rd }}\right)$ fractions of aqueous phases containing $1 \%$ sTMP GGM and BGX

292 The average molar mass value of the second and third fractions was in the order of $10^{6}-10^{9} \mathrm{~g} \mathrm{~mol}^{-1} \mathrm{suggesting}$ 293 these fractions were either large-sized soluble molecules or supramolecular aggregates or particles. To differentiate 294 macromolecular states of polysaccharides conformational plots are often used by plotting molar mass values as a 295 function of the radius of gyration $\left(\mathrm{R}_{g}\right)$. Slope values of $0.5-0.7$ and $>1$ usually exhibit polymer molecules in random coil and extended rod-like states, respectively and a slope of 0.33 or below indicates analytes of compact conformation-like spheres. (Podzimek 2011) Slope values of less than 0.33 were observed for both second and third fractions of sTMP GGM as well as for BGX (Fig. 2E) corroborating the fact that they were supramolecular instead of soluble molecules. These fractions could be dense aggregates or agglomerates resulting from the fusion of two or more interacting aggregates resembling supra-particles at the studied concentration of $1 \%$. Indeed, the absence of the third fraction at $0.2 \%$ concentration of these samples supported this assumption (Fig. S1). On the other hand, a conformational plot could not be established for the second fraction in BLN GGM. Because of its low MALS signal-to-noise ratio, reliable $\mathrm{R}_{g}$ values were not obtained suggesting that the supramolecular fraction was composed of a minor amount of assemblies/aggregates/dust particles. Conformational plots could also not be established for the first fractions as $\mathrm{R}_{g}$ values cannot be obtained due to the anisotropy of small-sized analytes (Podzimek 2011).

To understand the nanostructural characteristics of supramolecular fractions, SAXS experiments were performed

(Fig. 3). The samples were also studied after mechanical shearing treatment using a microfluidizer to observe any shear-induced nanostructural changes. Selected aqueous phases were also measured after being subjected to heating and freeze-thawing treatment. The scattering data from aqueous phases of sTMP GGM did not exhibit any correlation peak in the studied length scale $(2-200 \mathrm{~nm})$ with no difference in the scattering feature before and after high-shear treatment (Fig. 3A), and even after heating and freeze thawing (scattering data not shown). The purified extract from the TMP process (eTMP GGM) also exhibited similar features in SAXS. A similar observation was made in the BLN GGM sample (Fig. 3B). 
A

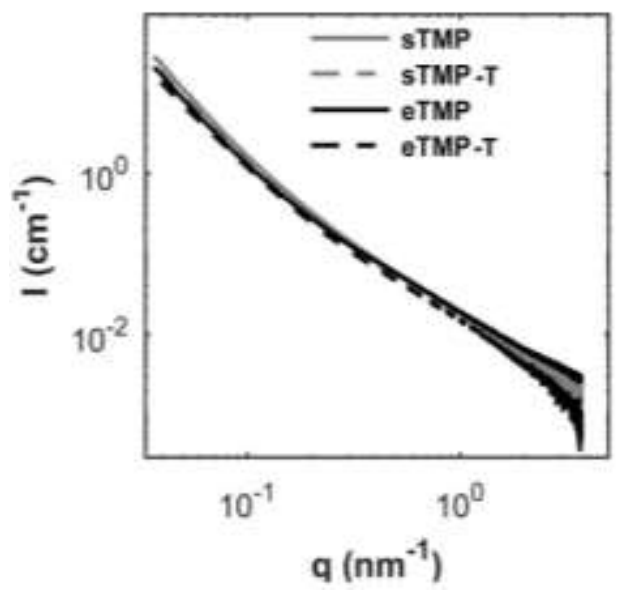

B

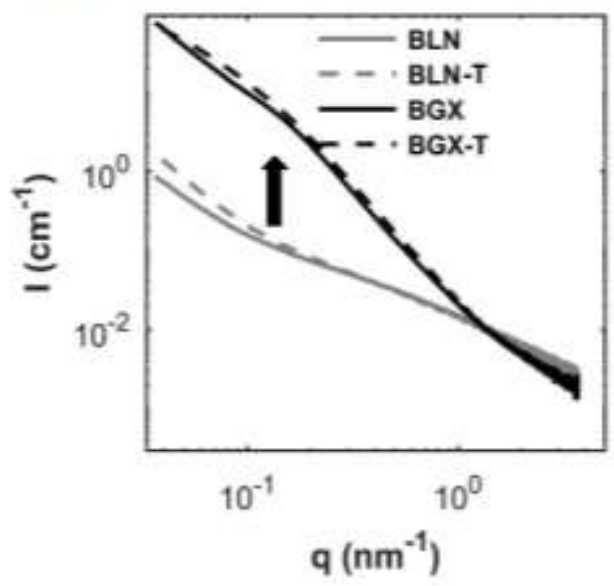

316 Fig. 3 Scattering intensity (I) as a function of scattering vector (q) from $1 \%$ (A) sTMP GGM, eTMP GGM, and

317 (B) BLN GGM and BGX before and after (indicated as -T in the legend) shear treatment

318 The scattering intensity of sTMP GGM and eTMP GGM scaled with a $p$-value of 2.81 and 2.73, respectively from

319 the power-law fitting (Equation 1) over a $q$-range of $0.04-0.1 \mathrm{~nm}^{-1}$ corresponding to a length scale of $60-160 \mathrm{~nm}$

320 in real space (Table S2). This obtained $p$-value indicated the fractal nature of aggregates with nearly 3D 321 morphology. The $p$-value reduced to $\approx 2.6$ after shear, heat, and freeze-thawing treatment indicating a small reduction in the packing density of aggregates. This corroborated the AF4 chromatograms of nominal changes in the shear-treated samples (Fig. S1A). At a smaller length scale, over a $q$-range of $0.26-1.05 \mathrm{~nm}^{-1}$ corresponding to a length scale of 6-20 nm, the eTMP GGM scaled with a $p$-value of 1.6-1.65. This is suggestive of rod-/disc-like objects, which are likely to be primary constituents of porous aggregates.

326 Conversely, the scattering intensity of BGX exhibited a broad correlation peak (Fig. 3B, indicated by an arrow in

327 B) with peak maxima corresponding to the length scale of $\approx 60 \mathrm{~nm}$. After shear treatment, the peak shifted slightly towards smaller $q$-values due to increased scattering objects from the shear-induced dissociation of large agglomerates, which was also observed in AF4 chromatograms (Fig. S1B). The scattering intensity at low $q$-region of $0.19-0.32 \mathrm{~nm}^{-1}$ corresponds to a length scale of 20-34 nm scaled with a $p$-value of 2.9-2.95 indicating mass

331 fractal 3D objects. The scattering intensity of BLN GGM was the lowest of all samples suggesting that scattering objects in the studied length scale were either smaller in size, lower in concentration, or less packed than the rest 333 of the samples. This supported our assumption of the loosely associated nature of the supramolecular fraction of BLN GGM. 
A combination of AF4-MALS and SAXS analyses provided comprehensive information on sample solubility and nanostructural characteristics of supramolecular fractions of wood hemicelluloses. To our knowledge, this is the

338 first study of this kind. Partial solubility of structurally close seed-derived galactomannans was reported earlier; however, conclusive evidence on the supramolecular fraction and their nanostructural information was not available (Gittings et al. 2000). On the other hand, the solubility comparison between wood-derived mannans and seed-derived mannans is not straightforward either because of variables such as degree of polymerization, substitution degree, and other non-polysaccharide components native to the source. Additionally, recovery processes are expected to change the interplay between these factors leading to differences in solubility of polysaccharides even from the same source. We have demonstrated this in the case of wood GGMs.

We observed significant solubility differences between industrially recovered GGMs from the TMP process (sTMP GGM and eTMP GGM) and hot water extracted BLN GGM. The former was largely insoluble with a supramolecular fraction with complex structural characteristics. The molar mass of the soluble fraction was also two times higher in GGMs from the TMP compared to the BLN process because of the higher degree of temperature-induced hydrolytic reactions during the hot water extraction process (Teo et al. 2010). Solubility differences between GGM from TMP and BLN process can be explained alone or in combination with differences in molar mass of soluble fraction, purity, and molecular structure. Naturally occurring lignin and lignin-derived aromatic compounds in GGMs, which are often considered impurities, can be another contributing factor to GGM solubility. We have previously observed aggregates and particles in crude hot water extracted GGMs using the

354 PHWE method (sPHWE GGM). These supramolecular fractions were removed largely when the extracts were purified using ethanol during the downstream process (ePHWE GGM). The absence of complex supramolecular fraction in BLN GGM, which had a small amount of phenolic content, supports the hypothesis. During the BLN process, extracts are recirculated multiple times to the biomass for the readsorption of extracted aromatic compounds (Schoultz 2015). Acidic or alkaline conditions employed during recovery processes also modify the substituents groups, namely acetyl, arabinose units, which are considered critical to wood hemicelluloses solubility

360 (Bosmans et al. 2014; Sjöström 1993; Xu et al. 2007). These substituents create steric hindrances preventing 361 intermolecular chain association and are relevant in uncharged polysaccharides like GGMs and BGX (see zeta 362 potential values in Table S4) (Dumitriu 2004). Further studies are required to elucidate the structure-solubility 363 relationship in GGMs.

364 In the next step, using the relatively insoluble GGMs from the TMP process (sTMP GGM and eTMP GGM), 365 soluble GGM from the BLN method (BLN GGM), and semi-soluble GGMs from PHWE (sPHWE GGM and 
366 ePHWE GGM), emulsions were prepared and examined for their interfacial structures and stability. Emulsions 367 from BGX and T20, a small molecular weight surfactant with higher surface activity than biopolymers were 368 included for comparison (Jafari et al. 2007).

\section{$369 \quad 3.3$ Emulsion study}

\section{$370 \quad$ 3.3.1 Interfacial structure of emulsions}

371 Interfacial properties such as, surface charge, thickness, and structure of emulsions play a key role in their physical 372 stability and other functionalities they are engineered for, e.g., drug and bioactive delivery, oxidative stability of 373 the lipid phase (McClements 2016). Adsorption of solid micro-/nanoparticles and their assembly at the oil-water 374 interface form a rigid disordered layer/network of particles (Ortiz et al. 2020) opposed to flexible viscoelastic layer 375 from soluble surface-active polymers (Wei et al. 2020). The type of interfacial structures in wood hemicelluloses376 stabilized emulsions have not been addressed so far due to lack of solubility data.

377 We investigated the interfacial structures of emulsions by combining SAXS (Fig. 4) with cryo-scanning electron 378 microscopy (Fig. 5). Scattering from emulsions comprises contributions from their aqueous phases (stabilizers + 379 buffer), oil, and oil/water interface. Scattering data from GGM-based emulsions did not exhibit any correlation 380 peak (Fig. 4A-C). The broad correlation peak in BGX and T20 emulsion in the length scale $\approx 40 \mathrm{~nm}$ (Fig. 4D) 381 likely represented supramolecular fractions of BGX as a similar observation was made in the aqueous phase of 382 BGX (Fig. 4B) and oil droplets of T20 emulsion, which were in a similar length scales (see droplet size data 383 further). 
A

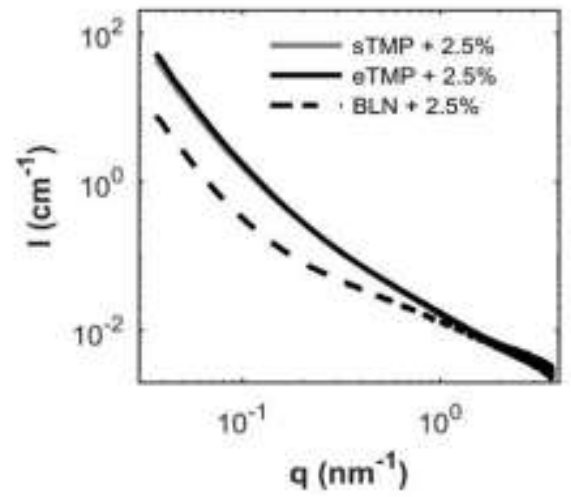

C

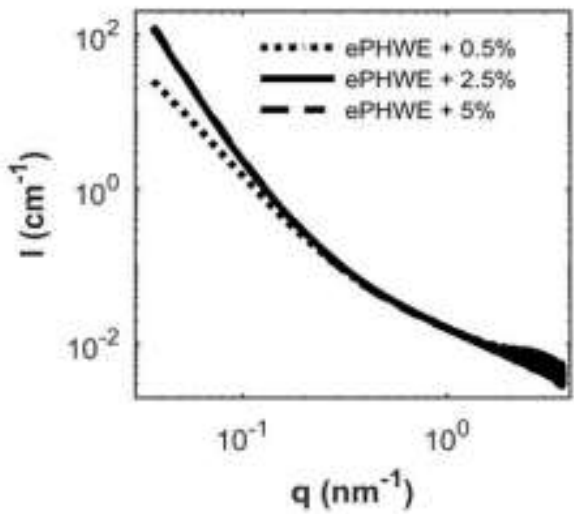

B

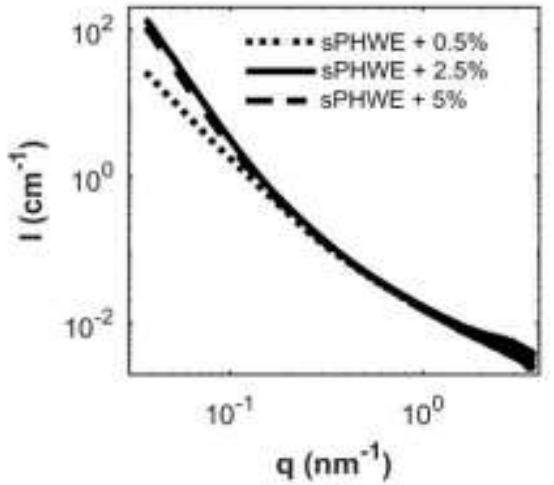

D

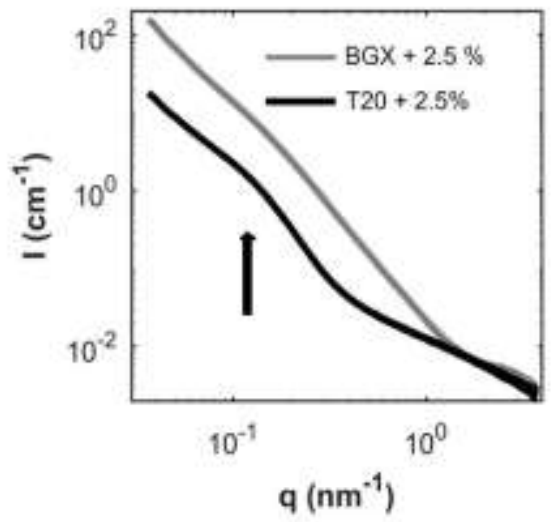

385 Fig. 4 Scattering intensity $(I)$ as a function of scattering vector $(q)$ of emulsions containing (A) sTMP GGM eTMP GGM and BLN GGM (B), sPHWE GGM (C), ePHWE GGM (D), and BGX and T20. All emulsions contained 1 wt $\%$ of emulsifier and $2.5 \mathrm{wt} \%$ rapeseed oil. Emulsions from sPHWE GGM and ePHWE GGM containing 0.5 $\mathrm{wt} \%$ and $5 \mathrm{wt} \%$ rapeseed oil were also measured

At high $q$-region (i.e., smaller length scales), the scattering intensity was dominated by the aqueous phases and oil. A broad correlation peak with a maximum at $q \approx 2 \mathrm{~nm}^{-1}$ originated from the structural organization of triglyceride

391 molecules in oil (Salentinig et al. 2017). At low q-region (i.e., larger length scales) scattering intensity from emulsions deviated from their aqueous phases and exhibited a power-law behavior suggesting the fractal nature of 393 the scattering objects. The power-law coefficient, $p$-value, was obtained from a linear model (Equation 2) which

394 takes into account scattering contributions from aqueous phases and oil. There were differences in $p$-values 395 between emulsions indicating differences in the structural organization at oil-water interfaces (Table S3).

396 Emulsions from both sTMP GGM and eTMP GGM exhibited $p>4$, BLN GGM and ePHWE GGM exhibited $397 p \approx 4$, and BGX exhibited $p \approx 3$ indicating diffused, smooth, and heavy surface fractal characteristics of interfaces, 398 respectively. The first point to note is, oil-water interface from stabilizers with a highly aggregated supramolecular 
400 The second point to note is the distinction of the fractal oil-water interface of BGX emulsion from the rest.

401 Scattering data from sPHWE GGM and T20 emulsions could not be fitted as their aqueous phases had correlation 402 peaks that were not present in the same length scale as in their emulsions, therefore, their subtraction was not 403 possible. The correlation peak in the aqueous phases of sPHWE GGM represented colloidal aggregates/lignin-rich 404 nanoparticles,(Bhattarai et al. 2020) whereas, in T20, it represented T20 micelles in the length scale of $\approx 4 \mathrm{~nm}$ 405 (data not shown) (Pal et al. 2015).

406 The theory of diffused interfaces with $p$-value > 4 observed in STMP GGM and eTMP GGM emulsions have not 407 been explored in detail, however, it is interpreted as structures transient with the length scale (Beaucage 1995). A 408 closer look at the sTMP GGM emulsion interface under cryo-SEM revealed white, dense, and compact objects of 409 100-200 nm size around the oil droplets (Fig. 5, 1a-c), very likely the supramolecular fraction discussed earlier. 410 Further, similar objects of varying morphology such as rod-or platelet-like were visualized in the continuous phase 411 of this emulsion (Fig. 5, 1d). This organization of supramolecular structures of different sizes and shapes in the 412 interfacial/near-interfacial regions is the proposed explanation behind the diffused interfaces in these emulsions. 413 In slightly soluble GGMs, sPHWE GGM, and ePHWE GGM, the surface of oil droplets appeared smooth (Fig. 5, 414 2a-c, 3a-c) most likely from the adsorption of soluble fraction. This agreed with the $p$-value obtained from SAXS 415 and a similar explanation is proposed for the emulsion from soluble BLN GGM. Some colloidal particles 416 presumably along with aggregates visualized as thin strips were observed in the continuous phase of sPHWE GGM 417 and ePHWE emulsions (Fig. 5, 2d and 3d). These fractions are expected to confer additional stability to emulsions 418 (discussed further). 

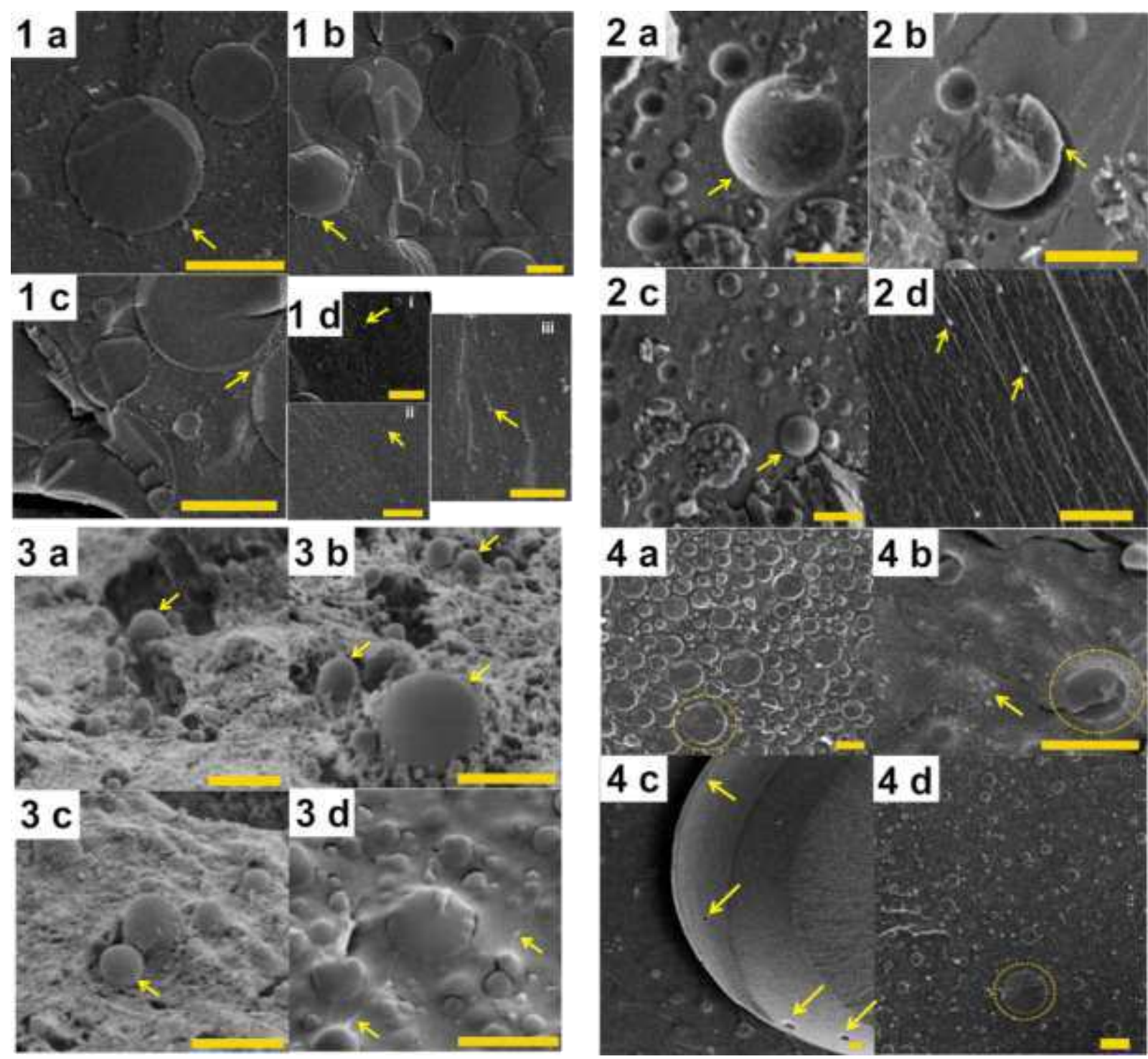

Fig. 5 Cryo-SEM images of emulsions each containing 1 wt\% (1 a-d) sTMP GGM, (2 a-d) sPHWE GGM, (3 a-d) ePHWE GGM, and (4 a-d) BGX and $2.5 \mathrm{wt} \%$ rapeseed oil. Scalebar- $1 \mu \mathrm{m}$

On the other hand, BGX with a comparably sophisticated supramolecular fraction as sTMP GGM vastly differed in interfacial characteristics (Fig. 5, panel 4a-d). Under SEM, white, dense, and mostly spherical objects were observed. These structures appeared to be concentrated at the oil/water interface (Fig. 5, panels 4a and 4d, dashed circle in panel 4b), which validates the SAXS findings of the heavy surface fractal nature of this emulsion interface. Some dents were observed on the surface of oil droplets (Fig. 5, panel 4c) indicating the adsorption of BGX

427 particles.

\subsubsection{Emulsion stability}

429 Surface-active soluble molecules and colloidal supramolecular particles adsorb at the interface, reduce interfacial 430 tension thereby, aiding the dispersion of oil to fine droplets and stabilizing them against coalescence. These 431 fractions in the unadsorbed state also create hindrance and aid to the stabilization by either forming viscoelastic 
432 gel and/or colloidal network. In our study, the average size of dispersed oil droplets and time-dependent changes 433 were evaluated during a 2-week storage time (Fig. 6A-B). The dynamic changes from droplets' 434 coalescence/flocculation in emulsions during 4-week storage were compared using the Turbiscan Stability Index 435 (TSI) (Fig. 6C-D).
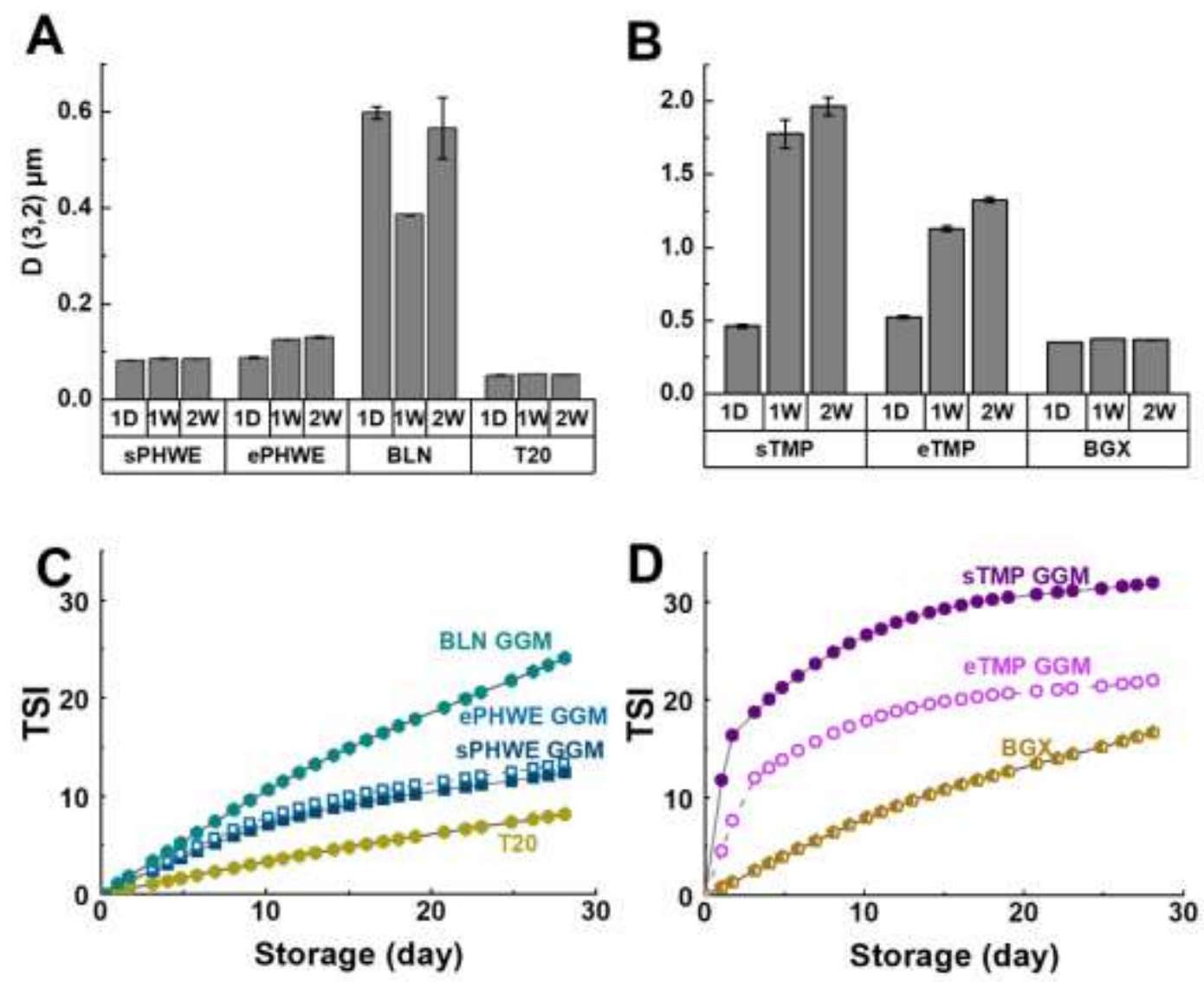

Fig. 6 (A, B) Surface-average diameter D(3,2) of emulsions each containing $2.5 \mathrm{wt} \%$ rapeseed oil and 1 wt $\%$ stabilizer measured on the preparation day (1D), after 1 week (1W), and 2 weeks (2W) of storage at RT. Note different scales between panels. (C, D) Evolution of Turbiscan stability index (TSI) in emulsions during four weeks of storage

441 Between wood hemicelluloses, the semi-soluble GGMs, sPHWE GGM, and ePHWE GGM, produced emulsions 442 with the lowest average droplet size that remained stable during the experimental storage period. The Turbiscan 443 Stability Index (TSI) values at the end of storage were lower suggesting fewer dynamic changes in emulsions (Fig. 444 6C). Emulsions' average droplet size was below $100 \mathrm{~nm}$ and even comparable with the surfactant T20. Surfactants 445 are highly surface-active because of hydrophilic and hydrophobic groups and low molecular weight. Similar 446 behavior exhibited by these GGMs supports the domination of surface-active soluble fraction of these samples 447 during interfacial adsorption, therefore, validates the findings of smooth interface from SAXS and cryo-SEM data. 448 However, emulsion from the soluble BLN GGM with a similar interfacial feature was unstable. It even showed 
some oiling-off immediately after the preparation; therefore, the presented droplet size results in Fig. 6A only provide partial information of droplet size. Lignin-derived phenolic compounds provide amphiphilicity to the GGMs (Lehtonen et al. 2018; Lehtonen et al. 2016). The poor performance of BLN GGM, which had the least phenolic content, indicates the importance of these compounds for interface stabilization. Having supramolecular fractions possibly confer additional stability by creating barriers.

Oil droplets in emulsions from insoluble GGMs, sTMP GGM and eTMP GGM were relatively larger. The final droplet size is defined by the adsorption kinetics of the emulsifiers, meaning small, surface-active molecules generally yield fine and small-sized droplets (Tadros 2017) compared to colloidal particles because of their high adsorption energy (Sarkar and Dickinson 2020). However, the stability of oil droplets in these emulsions was poor indicating insufficient interfacial coverage. BGX, on the other hand, exhibited good emulsification and stabilization ability. This suggests differences in hydrophobicity of supramolecular fractions between GGMs and BGX. Partial crystallinity has been reported in BGX;(Bosmans et al. 2014) therefore, it is likely that these BGX particles anchor the interface via crystalline hydrophobic segments.

462 The evolution of TSI values was fitted using a non-linear regression model to obtain $T S I_{\max }$, the maximum TSI 463 value reachable at infinite time and $k$, the rate constant of the dynamic changes in emulsions (Equation 3). Based 464 on $T^{2} I_{\max }$ values (Table 2), emulsions from semi-soluble GGMs (sPHWE GGM and ePHWE GGM) would be 465 physically stable for a long time followed by insoluble GGMs from the TMP process. The least stable emulsion 466 would be from soluble BLN GGM. The rate constant ( $k$ ) values suggest rapid alterations in emulsions from insoluble GGMs (sTMP GGM and eTMP GGM) before attaining a steady state as opposed to slow and progressive changes in emulsion from soluble BLN GGM. This signifies the role of supramolecular fractions conferring additional stability as fillers in the aqueous phases of emulsions (Dickinson 2017). This is further supported by the lower $T S I_{\max }$ values of emulsions from semi-soluble sPHWE GGM and ePHWE GGM compared to emulsion from small molecular weight surfactant T20. 
Table 2. Estimated model parameters; $T S I_{\max }, k$ with standard error of estimated parameters, and $\mathrm{R}^{2}$ obtained using Equation 3 on TSI of emulsions each containing $1 \mathrm{wt} \%$ hemicellulose, T20, and $2.5 \mathrm{wt} \%$ rapeseed oil.

\begin{tabular}{|c|c|c|c|c|c|c|c|}
\hline & \multicolumn{3}{|c|}{ Soluble to semi-soluble } & \multicolumn{3}{|c|}{ Insoluble } & \multirow{2}{*}{$\begin{array}{c}\text { Surfactant } \\
\mathrm{T} 20\end{array}$} \\
\hline & $\begin{array}{l}\text { BLN } \\
\text { GGM }\end{array}$ & $\begin{array}{c}\text { ePHWE } \\
\text { GGM }\end{array}$ & $\begin{array}{c}\text { sPHWE } \\
\text { GGM }\end{array}$ & sTMP GGM & eTMP GGM & BGX & \\
\hline$T S I_{\max }$ & $\begin{array}{c}48.33 \pm \\
1.02^{\mathrm{c}}\end{array}$ & $15.58 \pm 0.23^{\mathrm{d}}$ & $14.76 \pm 0.22^{\mathrm{d}}$ & $30.15 \pm 0.50^{\mathrm{a}}$ & $20.86 \pm 0.21^{\mathrm{b}}$ & $26.85 \pm 0.50^{\mathrm{e}}$ & $24.14 \pm 0.69^{f}$ \\
\hline$k$ & $0.02 \pm 0.00^{c}$ & $0.07 \pm 0.02^{\mathrm{d}}$ & $0.06 \pm 0.00^{\mathrm{d}}$ & $0.27 \pm 0.02^{\mathrm{a}}$ & $0.22 \pm 0.01^{\mathrm{b}}$ & $0.03 \pm 0.00^{\mathrm{c}, \mathrm{d}}$ & $0.01 \pm 0.00^{c}$ \\
\hline $\mathbf{R}^{2}$ & $>0.99$ & $>0.99$ & $>0.99$ & 0.94 & 0.98 & $>0.99$ & $>0.99$ \\
\hline
\end{tabular}

Estimated parameters are different than $0(P<0.05)$.

4 Conclusion

The present study demonstrated that wood hemicelluloses exist in various macromolecular states affecting their functionality in emulsions. GGMs obtained from different recovery approaches that are of current interest were found to modify the solubility of polysaccharides. GGMs existed as a soluble fraction with a molar mass of $1.0-$ $2.0 \times 10^{4} \mathrm{~g} \mathrm{~mol}^{-1}$ and in supramolecular fractions with aggregates and agglomerates. The macromolecular state of polysaccharides affected interfacial structures and emulsion stability. The supramolecular fraction affected the interfacial morphology via an interplay between their affinities for interfacial adsorption and their share in the sample. When hemicelluloses in molecular form with a high interfacial adsorption affinity dominated, smooth interfaces were observed. When supramolecular fractions dominated, diffused (sTMP GGM and eTMP GGM) or heavy surface fractal features (BGX) were observed depending on their packing at the interface/near-interface. The supramolecular fraction contributed to the emulsion stability by creating interfacial barriers. The obtained findings suggest that semi-solubility improves the performance of biopolymers in oil-in-water systems instead of full solubility and demonstrate biorefinery techniques as efficient strategies to tailor polysaccharides' functionality.

\section{Acknowledgements}

496 The doctoral program of Food Chain and Health at the University of Helsinki and EU-COST Action FP1306 are 497 acknowledged for funding MB and her scientific visit to the University of Natural Resources and Life Sciences 498 (BOKU), Austria for AF4 measurement. The Väisälä Fund is acknowledged for funding the travel of Inkeri Kontro 499 to Diamond Light Source Synchrotron in the UK. Dr. Petri Kilpeläinen from Natural Resources Institute Finland 500 (Luke), and Professor Stefan Willför, and Professor Chunlin Xu from Åbo Akademi University, Finland are 501 acknowledged for providing GGM samples. Dr. Katsuaki Inoe and B21 beamline scientists (Robert Rambo and 502 Nikul Khunti) at the Diamond Synchrotron Facility, UK, are acknowledged for their assistance during SAXS 
503

504

505

506

507

508

509

510

511

512

513

514

515

516

517

518

519

520

521

522

523

524

525

526

527

528

529

530

531

532

533

534

535

536

537

538

539

measurement. We also acknowledge the Core Facility for Integrated Microscopy, Faculty of Health and Medical Sciences, University of Copenhagen, Denmark for Cryo-SEM imaging services. Hongbo Zhao and Troy Faithfull from the University of Helsinki, Finland is acknowledged for assistance during emulsion characterization and manuscript editing, respectively.

\section{Ethics declarations}

There are no conflicts of interests to declare.

\section{References}

Beaucage G (1995) Approximations Leading to a Unified Exponential/Power-Law Approach to Small-Angle Scattering. J. Appl. Crystallogr. 28 (6): 717-728. https://doi.org/10.1107/S0021889895005292

Bhattarai M, Pitkanen L, Kitunen V, Korpinen R, Ilvesniemi H, Kilpelainen PO, Lehtonen M, Mikkonen KS (2019) Functionality of spruce galactoglucomannans in oil-in-water emulsions. Food Hydrocoll. 86: 154-161. https://doi.org/10.1016/j.foodhyd.2018.03.020

Bhattarai M, Sulaeva I, Pitkänen L, Kontro I, Tenkanen M, Potthast A, Mikkonen KS (2020) Colloidal features of softwood galactoglucomannans-rich extract. Carbohydr. Polym. 241: 116368. https://doi.org/10.1016/j.carbpol.2020.116368

Bosmans TJ, Stépán AM, Toriz G, Renneckar S, Karabulut E, Wågberg L, Gatenholm P (2014) Assembly of Debranched Xylan from Solution and on Nanocellulosic Surfaces. Biomacromolecules 15 (3): 924-930. https://doi.org/10.1021/bm4017868

Dickinson E (2003) Hydrocolloids at interfaces and the influence on the properties of dispersed systems. Food Hydrocoll. 17 (1): 25-39. https://doi.org/10.1016/S0268-005X(01)00120-5

Dickinson E (2017) Biopolymer-based particles as stabilizing agents for emulsions and foams. Food Hydrocoll. 68: 219-231. https://doi.org/10.1016/j.foodhyd.2016.06.024

Dumitriu S (2004) Polysaccharides: structural diversity and functional versatility.2. Ed. CRC press, Boca Raton, FL, USA.

Ebringerova A, Hromadkova Z, Burchard W, Dolega R, Vorwerg W (1994) Solution properties of waterinsoluble rye-bran arabinoxylan. Carbohydr. Polym. 24 (3): 161-169. https://doi.org/10.1016/0144$\underline{8617(94) 90126-0}$

Gittings MR, Cipelletti L, Trappe V, Weitz DA, In M, Marques C (2000) Structure of Guar in Solutions of H2O and D2O: An Ultra-Small-Angle Light-Scattering Study. J. Phys. Chem. B 104 (18): 4381-4386. https://doi.org/10.1021/jp9943833

Jafari SM, He Y, Bhandari B (2007) Effectiveness of encapsulating biopolymers to produce sub-micron emulsions by high energy emulsification techniques. Food Res. Int. 40 (7): 862-873. https://doi.org/10.1016/j.foodres.2007.02.002

Kilpeläinen PO, Hautala SS, Byman OO, Tanner LJ, Korpinen RI, Lillandt MKJ, Pranovich AV, Kitunen VH, Willför SM, Ilvesniemi HS (2014) Pressurized hot water flow-through extraction system scale up from the laboratory to the pilot scale. Green Chem. 16 (6): 3186-3194.

http://dx.doi.org/10.1039/C4GC00274A 
Lam S, Velikov KP, Velev OD (2014) Pickering stabilization of foams and emulsions with particles of biological origin. Curr. Opin. Colloid Interface Sci. 19 (5): 490-500. https://doi.org/10.1016/j.cocis.2014.07.003

Lehtonen M, Merinen M, Kilpeläinen PO, Xu C, Willför S, Mikkonen KS (2018) Phenolic residues in spruce galactoglucomannans improve stabilization of oil-in-water emulsions. J. Colloid Interface Sci. 512: 536-547. https://doi.org/10.1016/j.jcis.2017.10.097

Lehtonen M, Teräslahti S, Xu C, Yadav MP, Lampi A-M, Mikkonen KS (2016) Spruce galactoglucomannans inhibit lipid oxidation in rapeseed oil-in-water emulsions. Food Hydrocoll. 58: 255-266. https://doi.org/10.1016/j.foodhyd.2016.03.006

Linder Å, Bergman R, Bodin A, Gatenholm P (2003) Mechanism of Assembly of Xylan onto Cellulose Surfaces. Langmuir 19 (12): 5072-5077. https://doi.org/10.1021/la0341355

McClements DJ (2016) Food emulsions : principles, practices, and techniques.3. Ed. CRC Press, Boca Raton, London, New York.

Mikkonen KS, Kirjoranta S, Xu C, Hemming J, Pranovich A, Bhattarai M, Peltonen L, Kilpeläinen P, Maina N, Tenkanen M et al. (2019) Environmentally-compatible alkyd paints stabilized by wood hemicelluloses. Ind Crops Prod 133: 212-220. https://doi.org/10.1016/j.indcrop.2019.03.017

Mikkonen KS, Merger D, Kilpeläinen P, Murtomäki L, Schmidt US, Wilhelm M (2016a) Determination of physical emulsion stabilization mechanisms of wood hemicelluloses via rheological and interfacial characterization. Soft Matter 12 (42): 8690-8700. http://dx.doi.org/10.1039/C6SM01557C

Mikkonen KS, Xu C, Berton-Carabin C, Schroën K (2016b) Spruce galactoglucomannans in rapeseed oil-inwater emulsions: Efficient stabilization performance and structural partitioning. Food Hydrocoll. 52: 615-624. https://doi.org/10.1016/j.foodhyd.2015.08.009

Nakauma M, Funami T, Noda S, Ishihara S, Al-Assaf S, Nishinari K, Phillips GO (2008) Comparison of sugar beet pectin, soybean soluble polysaccharide, and gum arabic as food emulsifiers. 1. Effect of concentration, pH, and salts on the emulsifying properties. Food Hydrocoll. 22 (7): 1254-1267. https://doi.org/10.1016/j.foodhyd.2007.09.004

Ortiz DG, Pochat-Bohatier C, Cambedouzou J, Bechelany M, Miele P (2020) Current Trends in Pickering Emulsions: Particle Morphology and Applications. Engineering 6 (4): 468-482. https://doi.org/10.1016/j.eng.2019.08.017

Pal A, Deenadayalu N, Chaudhary S (2015) Effect of hydrophilic ionic liquid on the micellar properties of aqueous Tween-20. Fluid Phase Equilib. 391: 67-71. https://doi.org/10.1016/j.fluid.2015.02.005

Podzimek S (2011) Light Scattering, Size Exclusion Chromatography and Asymmetric Flow Field Flow Fractionation. Wiley, New Jersey, USA, Canada.

Salentinig S, Amenitsch H, Yaghmur A (2017) In Situ Monitoring of Nanostructure Formation during the Digestion of Mayonnaise. ACS Omega 2 (4): 1441-1446. https://doi.org/10.1021/acsomega.7b00153

Sanchez C, Nigen M, Mejia Tamayo V, Doco T, Williams P, Amine C, Renard D (2018) Acacia gum: History of the future. Food Hydrocoll. 78: 140-160. https://doi.org/10.1016/j.foodhyd.2017.04.008

Sarkar A, Dickinson E (2020) Sustainable food-grade Pickering emulsions stabilized by plant-based particles. Curr. Opin. Colloid Interface Sci. 49: 69-81. https://doi.org/10.1016/j.cocis.2020.04.004

Schmidt PW (1991) Small-angle scattering studies of disordered, porous and fractal systems. J. Appl. Crystallogr. 24 (5): 414-435. https://doi.org/10.1107/S0021889891003400 
Sjöström E (1993) Wood chemistry: fundamentals and applications.2. Ed. Gulf professional publishing, San Diego, California, USA.

Tadros TF (2017) Basic Principles of Interface Science and Colloid Stability. De Gruyter, Berlin Boston.

Teleman A, Tenkanen M, Jacobs A, Dahlman O (2002) Characterization of O-acetyl-(4-Omethylglucurono)xylan isolated from birch and beech. Carbohydr. Res. 337 (4): 373-377. https://doi.org/10.1016/S0008-6215(01)00327-5

Teo CC, Tan SN, Yong JWH, Hew CS, Ong ES (2010) Pressurized hot water extraction (PHWE). J. Chromatogr. 1217 (16): 2484-2494. https://doi.org/10.1016/j.chroma.2009.12.050

Thornton J (1994) Polysaccharides dissolved from Norway spruce in thermomechanical pulping and peroxide bleaching J. Wood Chem. Technol. 14 (2): 159-175. https://doi.org/10.1080/02773819408003092 Centrifugal fractionation of softwood extracts improves biorefinery workflow and yields functional emulsifiers. Green Chem. 21: 4691-4705. http://dx.doi.org/10.1039/C9GC02007A

Wei Y, Xie Y, Cai Z, Guo Y, Wu M, Wang P, Li R, Zhang H (2020) Interfacial and emulsion characterisation of chemically modified polysaccharides through a multiscale approach. J. Colloid Interface Sci. 580: 480492. https://doi.org/10.1016/j.jcis.2020.07.048

Whistler RL. Solubility of Polysaccharides and Their Behavior in Solution. In: Isbell HS, editor. Carbohydrates in Solution. American Chemical Society, Washington, DC, USA 1973: 242-255.

600

Willför S, Rehn P, Sundberg A, Sundberg K, Holmbom B (2003) Recovery of water-soluble

601 acetylgalactoglucomannans from mechanical pulp of spruce. Tappi J. 2 (11): 27-32.

Xu C, Willfor S, Sundberg K, Pettersson C, Holmbom B (2007) Physico-chemical characterization of spruce galactoglucomannan solutions: stability, surface activity and rheology. Cellul. Chem. Technol. 41 (1):

604 51.

605

Zhang L, Shi Z, Shangguan W, Fang Y, Nishinari K, Phillips GO, Jiang F (2015) Emulsification properties of 606 sugar beet pectin after modification with horseradish peroxidase. Food Hydrocoll. 43: 107-113. 


\section{Supporting Information}

619 Solubility of spruce galactoglucomannans determines interfacial morphology and 620 emulsion stability

621 Mamata Bhattarai $^{{ }^{*}}{ }^{*}$, Inkeri Kontro $^{2}$, Irina Sulaeva $^{3}$, Fabio Valoppi $^{1,4}$, Antje Potthast $^{3}$, Kirsi S. Mikkonen ${ }^{1,4}$

$622{ }^{1}$ Department of Food and Nutrition, P.O. Box 66, 00014 University of Helsinki, Finland

$623{ }^{2}$ Department of Physics, P.O. Box 64, 00014 University of Helsinki, Finland

$624{ }^{3}$ Department of Chemistry, University of Natural Resources and Life Sciences, Konrad-Lorenz-Straße. 24, 3430

625 Tulln, Austria

$626{ }^{4}$ Helsinki Institute of Sustainability Science, University of Helsinki, P.O. Box 65, Finland

627 Corresponding author: mamata.bhattarai@helsinki.fi

\section{A. Asymmetrical flow field-flow fractionation}

629 Table S1. Fitted weight-average molar mass value of seprarated fractions of $1 \%$ hemicelluloses in $25 \mathrm{mM}$ sodium 630 citrate buffer at $\mathrm{pH} 4.5$ analyzed without filtration (unfiltered) and filtered with $0.2 \mu \mathrm{m}$ filter pore (filtered) and 631 total sample recovery of unfiltered samples.

\begin{tabular}{|c|c|c|c|c|c|c|c|}
\hline \multirow{3}{*}{ Samples } & \multicolumn{6}{|c|}{ Molar mass [g mol$\left.{ }^{-1}\right]$} & \multirow{3}{*}{$\begin{array}{c}\text { Total sample } \\
\text { recovery } \%\end{array}$} \\
\hline & \multicolumn{2}{|c|}{$1^{\text {st }}$ fraction } & \multicolumn{2}{|c|}{$2^{\text {nd }}$ fraction } & \multicolumn{2}{|c|}{$3^{\text {rd }}$ fraction } & \\
\hline & unfiltered & filtered & unfiltered & filtered & unfiltered & filtered & \\
\hline sTMP GGM & $2.4 \times 10^{5}$ & $2.1 \times 10^{4}$ & $2.3 \times 10^{6}$ & $1.2 \times 10^{6}$ & $6.7 \times 10^{9}$ & $4.9 \times 10^{8}$ & 68.6 \\
\hline BLN GGM & $8.9 \times 10^{3}$ & $1.0 \times 10^{4}$ & $1.2 \times 10^{6}$ & $5.9 \times 10^{4}$ & $\mathrm{n} / \mathrm{a}$ & $\mathrm{n} / \mathrm{a}$ & 58.5 \\
\hline BGX & $3.6 \times 10^{4}$ & $1.5 \times 10^{4}$ & $1.3 \times 10^{7}$ & $1.4 \times 10^{7}$ & $1.0 \times 10^{9}$ & $4.3 \times 10^{8}$ & 55.7 \\
\hline
\end{tabular}

632

$\mathrm{n} / \mathrm{a}=$ not applicable

633 Refer to Fig. 2 for information about fractions. Zimm formalism was used for molar mass calculation of the first

634 fraction and Berry for the second and third fraction (when applicable). 
A

B
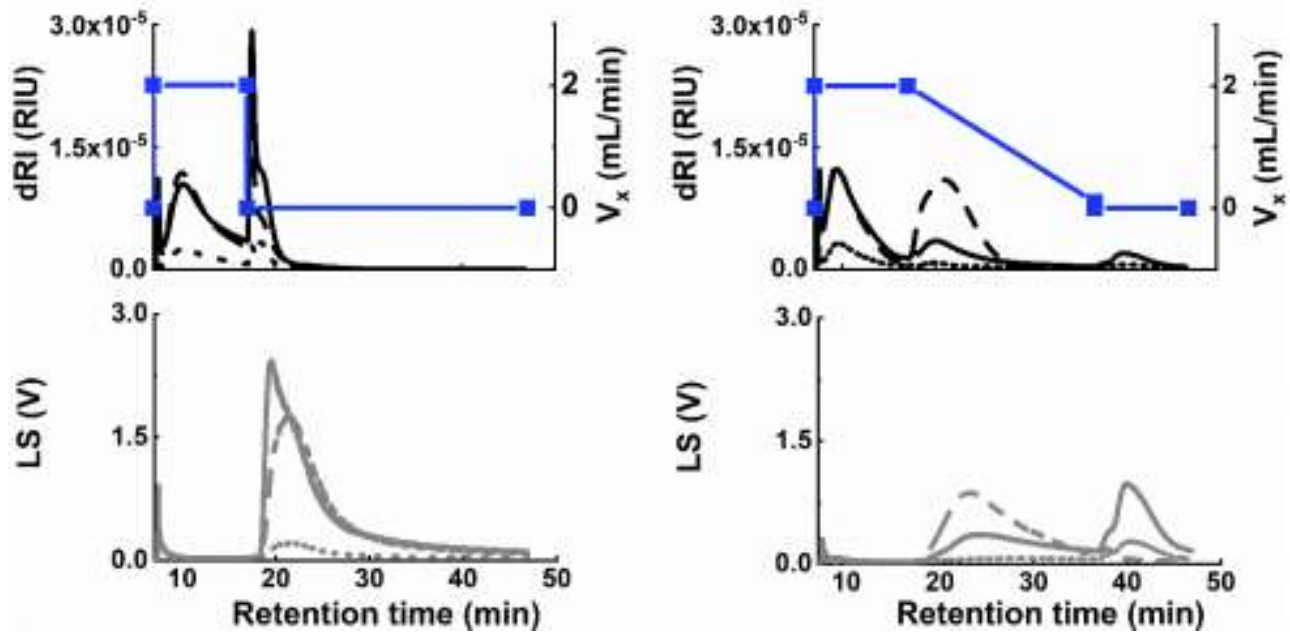

637 Fig. S1. Differential refractive index (dRI) and light scattering (LS) signals in refractive index units and Volt from

638 AF4 elution of (A) sTMP GGM and (B) BGX, both at $0.2 \%$ concentration (dotted line), $1 \%$ before shear treatment 639 (solid line) and after shear treatment (dashed line). The samples were mixed in $25 \mathrm{mM}$ sodium citrate buffer at $\mathrm{pH}$

640 4.5. A $350 \mu \mathrm{m}$ spacer was used and applied crossflow rate $\left(\mathrm{V}_{\mathrm{x}}\right)$ during elution is presented with dRI signal.

\section{B. Small-angle X-ray scattering}

642 Table S2. Power-law coefficient $(p)$ of the $1 \%$ aqueous phases containing sTMP GGM, eTMP GGM, and BGX.

643 sTMP GGM and eTMP GGM were measured before (untreated) and after shear treatment (treated), after heating 644 to $70{ }^{\circ} \mathrm{C}$ (heated), and freeze-thawing (thawed) treatment.

\begin{tabular}{lcccc}
\hline Samples & $p_{\text {untreated }}$ & $p_{\text {treated }}$ & $\begin{array}{c}q \text { range } \\
{\left[\mathrm{nm}^{-1}\right]}\end{array}$ & Size scale [nm] \\
\hline sTMP GGM & 2.81 & 2.69 & $0.04-0.1$ & $60-160$ \\
\hline sTMP GGM-heated & 2.66 & 2.64 & $0.04-0.1$ & $60-160$ \\
\hline sTMP GGM-thawed & 2.69 & 2.68 & $0.04-0.1$ & $60-160$ \\
\hline eTMP GGM & $2.73 ; 1.60$ & $2.59 ; 1.65$ & $0.04-0.1 ; 0.26-1.05$ & $60-160 ; 6-20$ \\
\hline eTMP GGM-heated & 2.66 & 2.48 & $0.04-0.1$ & $60-160$ \\
\hline eTMP GGM-thawed & 2.63 & 2.6 & $0.04-0.1$ & $60-160$ \\
\hline BGX & 2.95 & 2.90 & $0.19-0.32$ & $20-34$ \\
\hline
\end{tabular}



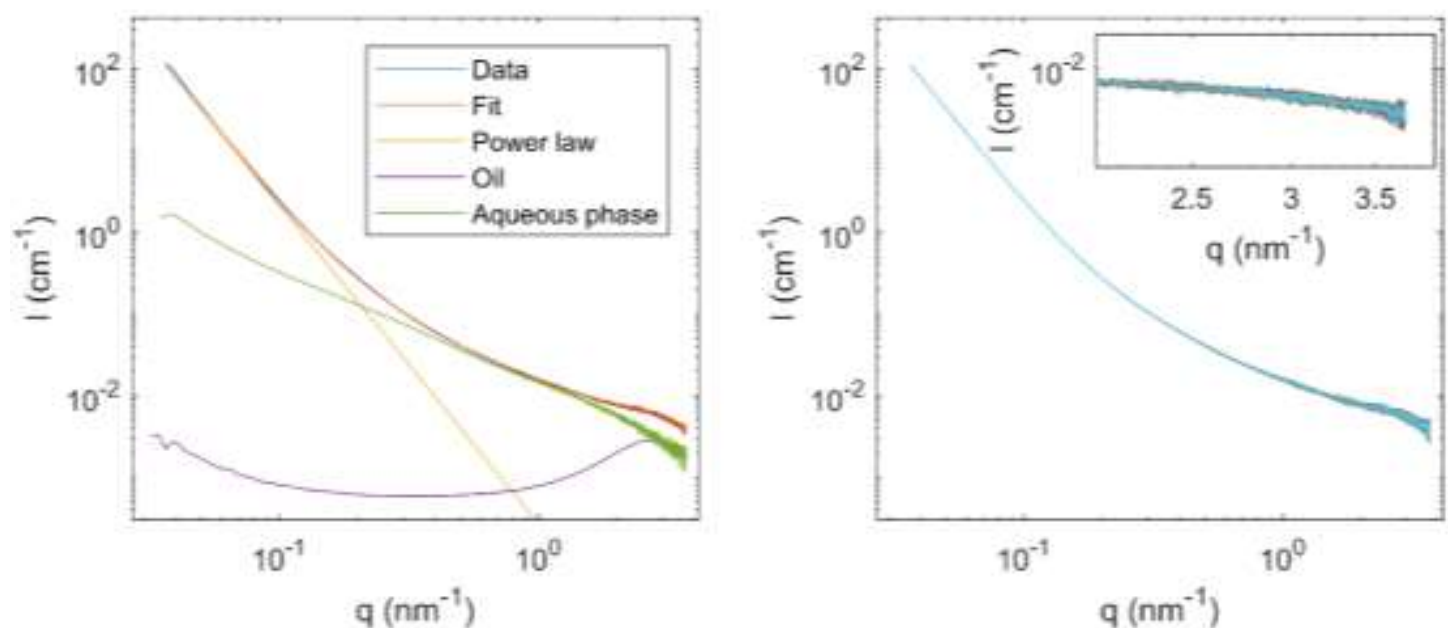

645 Fig. S2. (A) An example of power-law fit on the scattering intensity of emulsion containing $1 \mathrm{wt} \%$ ePHWE GGM 646 and $2.5 \mathrm{wt} \%$ rapeseed oil (Data). The scattering intensity of bulk rapeseed oil (Oil) and the aqueous phase containing $1 \%$ ePHWE GGM (Aqueous phase). (B) Example of convergence of fits to data sets simulated by MC procedure on the same emulsion. Inset: fits in high $q$-region.

Table S3. Power law fitting coefficient $(p)$ and standard deviation of fitting parameters reported as fitting error from emulsions each containing $1 \mathrm{wt} \%$ sTMP GGM, eTMP GGM, BLN GGM, ePHWE GGM, and BGX, and 2.5 $\mathrm{wt} \%$ rapeseed oil. The ePHWE GGM was also studied with $0.5 \mathrm{wt} \%$ and $5 \mathrm{wt} \%$ oil content and stored for 8 weeks $654(8 \mathrm{~W})$

\begin{tabular}{llll}
\hline Samples & Oil content $\%$ & $p$ & $\begin{array}{l}\text { Fitting } \\
\text { error }\end{array}$ \\
\hline sTMP GGM & 2.5 & -4.9436 & 0.0088 \\
\hline eTMP GGM & 2.5 & -4.3799 & 0.007 \\
\hline BLN GGM & 2.5 & -3.7993 & 0.0046 \\
\hline & 0.5 & -3.2738 & 0.0016 \\
ePHWE GGM & 2.5 & -3.8996 & 0.0019 \\
& $2.58 \mathrm{~W}$ & -3.9042 & 0.002 \\
& 5 & -4.0057 & 0.0015 \\
\hline BGX & 2.5 & -2.918 & 0.0036 \\
\hline
\end{tabular}




\section{Zeta potential}

659 Table S4. $\zeta$-potential $(\mathrm{mV})$ of aqueous phases containing $1 \%$ hemicelluloses in $25 \mathrm{mM}$ sodium citrate buffer at $660 \mathrm{pH}$ 4.5. Results are mean values of the $\zeta$-potential and standard error of the mean $(\mathrm{n}=3)$.

\begin{tabular}{ll}
\hline Samples & $\zeta$-potential $[\mathrm{mV}]$ \\
\hline sTMP GGM & $-8.57 \pm 0.36$ \\
\hline eTMP GGM & $-7.51 \pm 0.04$ \\
\hline BLN GGM & $-2.55 \pm 0.08$ \\
\hline sPHWE GGM & $-2.80 \pm 0.22$ \\
\hline ePHWE GGM & $-6.87 \pm 0.98$ \\
\hline BGX & $-15.8 \pm 0.91$ \\
\hline
\end{tabular}

661

\section{Droplet size distribution of emulsions}
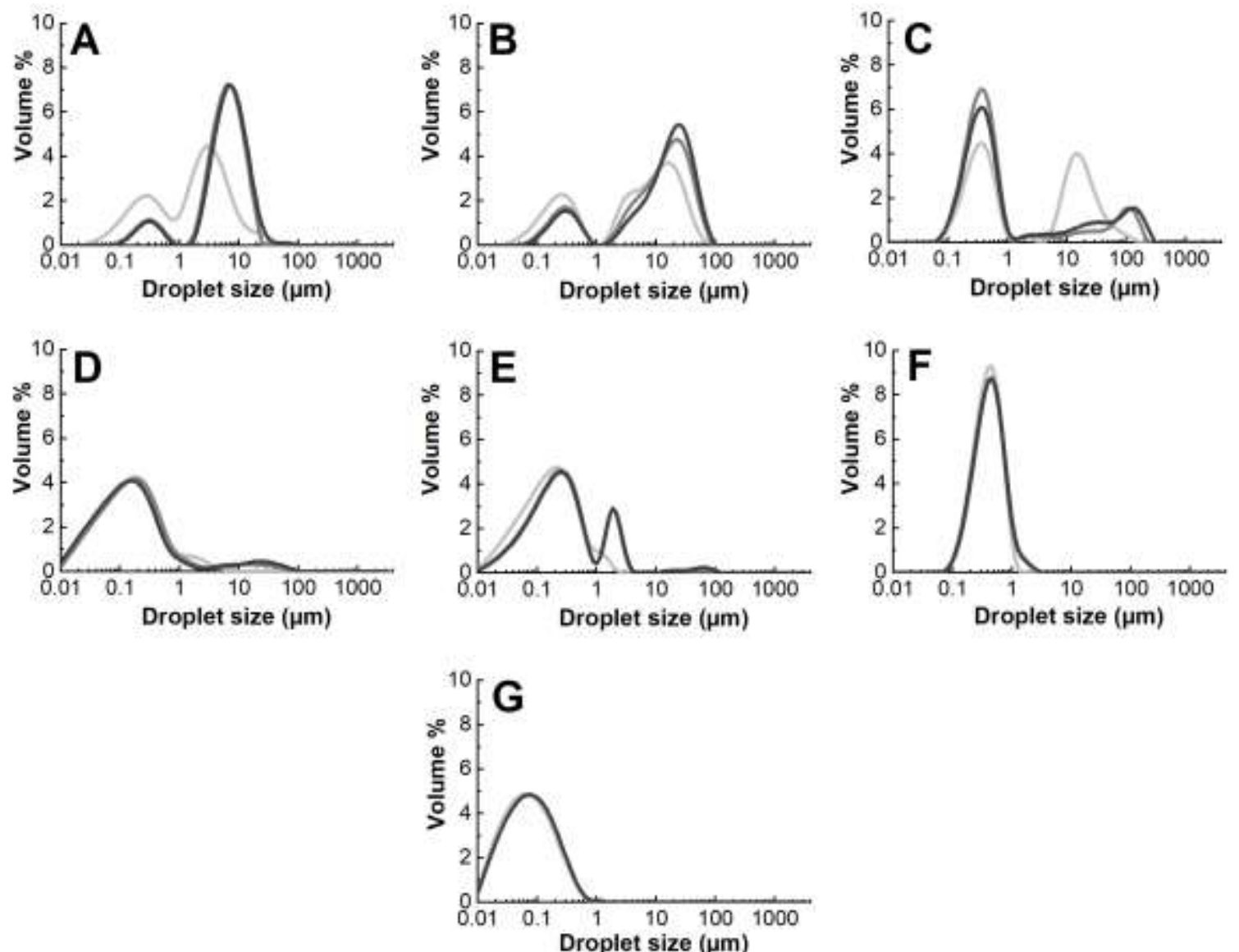

664 Fig. S3. Droplet size distributions of emulsions containing $2.5 \mathrm{wt} \%$ rapeseed oil stabilized by $1 \mathrm{wt} \%$ (A) sTMP 665 GGM (B) eTMP GGM (C) BLN GGM (D) sPHWE GGM (E) ePHWE GGM (F) BGX (G) T20 measured on the 666 preparation day, after one week and two weeks of storage at RT. Color darkening of lines indicates increasing 667 storage time of emulsions. 